\title{
Thread-modular Analysis of Release-Acquire Concurrency
}

\author{
Divyanjali Sharma ${ }^{1}$ and Subodh Sharma ${ }^{1}$ \\ Indian Institute of Technology Delhi \{divyanjali, svs\}@cse.iitd.ac.in
}

\begin{abstract}
We present a thread-modular abstract interpretation (TMAI) technique to verify programs under the release-acquire (RA) memory model for safety property violations. The main contributions of our work are: we capture the execution order of program statements as an abstract domain, and propose a sound upper approximation over this domain to efficiently reason over RA concurrency. The proposed domain is general in its application and captures the ordering relations as a first-class feature in the abstract interpretation theory. In particular, the domain represents a set of sequences of modifications of a global variable in concurrent programs as a partially ordered set. Under the upper approximation, older sequenced-before stores of a global variable are forgotten and only the latest stores per variable are preserved. We establish the soundness of our proposed abstractions and implement them in a prototype abstract interpreter called PRIORI. The evaluations of PRIORI on existing and challenging RA benchmarks demonstrate that the proposed technique is not only competitive in refutation, but also in verification. PRIORI shows significantly fast analysis runtimes with higher precision compared to recent state-of-the-art tools for RA concurrency.
\end{abstract}

\section{Introduction}

We investigate the problem of verifying programs with assertions executing under the release-acquire (RA) fragment of the C11 standard [15] where every store is a release write and every load is an acquire read. The reachability problem under the RA model (with compare-and-swap) has been recently shown to be undecidable [1]. The model is described axiomatically and correctness of programs under the model is defined by acyclicity axioms, which can appear obscure.

Notwithstanding the undecidability result, RA model is still one of the cleaner subsets of the C11 standard with relatively well-behaved semantics and has been a subject of active study in recent times $[4,33,23,18,19,1]$. An incomplete but intuitive understanding of RA concurrency is usually provided through reorderings - the redordering of an acquire load (or release store) with any access that follow (or precede) it in program order is disallowed. The RA model indeed provides weaker guarantees than SC, which allows for the construction of high performance implementations (e.g., read-copy-update synchronisation [33]) without making programmability overly complex. 
However, as noted in [23], RA programs can produce counter-intuitive outcomes that are unexplainable via interleaving of instructions. Consider the example execution graph (or just execution) of a 4-threaded program (IRIW) in Figure 1. It shows through appropriate reads-from (rf), sequence-before (sb) and data/control dependency (dep) edges that the property $P$ can evaluate to false under RA model (i.e., $r 1=r 3=1, r 2=r 4=0$ ). However, when the execution is interpreted under interleaving execution semantics (such as in SC, TSO, and PSO), the property is evidently valid because of a to-

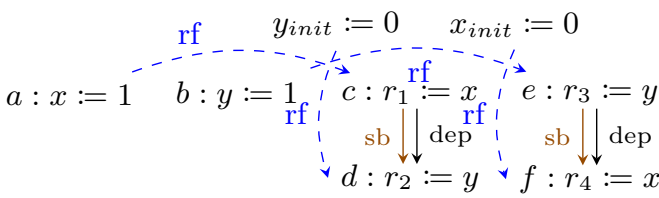

$$
\begin{aligned}
& P: r_{1}=1 \wedge r_{3}=1 \Longrightarrow r_{4} \neq 0 \wedge r_{2} \neq 0
\end{aligned}
$$

(IRIW)

Fig. 1: IRIW execution graph with reads-from (rf) and sequence-before (sb) edges tal ordering between $a$ and $b$ (i.e., $a$ before $b$ or vice-versa). Nonetheless, there are some fascinating aspects of RA semantics - (i) a total order on the stores of each global memory location (called the modification order) that restricts loads reading from overwritten stores, and (ii) when a load instruction of a thread $t$ observes (or synchronizes with) a store from another thread $t^{\prime}$, then all the prior stores observed by $t^{\prime}$ up to the synchronizing store also become observable to $t$. It is worth noting that this lack of immediate global visibility of updates, as mentioned in (ii) above, makes programs under RA semantics naturally amenable to localized or thread-modular reasoning, which is a well-considered area of research.

Thread-modular approaches are known to be sound for safety properties [13]. The basic idea behind thread-modular reasoning is to verify each thread separately with an environment assumption to model the effects of the execution of other threads. The environment assumption of each thread is usually specified by a relation (referred to as interference relation in this paper), which includes all the stores to global memory of other threads that may affect the loads of the thread. The analysis proceeds iteratively until for each thread the effects of its environment assumption on its operations reach a fix-point. As a model checking approach, they were first introduced for programs under SC semantics [10]. In the recent past, several thread-modular contributions [32, 26, 31, 21,22] have been presented in the context of verifying programs under weak memory models such as TSO, PSO and RMO. However, in our observation, prior proposals run into fundamental limitations when applying them to RA or other non-multicopyatomic memory models such as ARMv7 and POWER.

Techniques presented in $[31,32]$ model store buffers to analyze TSO and PSO programs. Evidently, RA program behaviors cannot be simulated using store buffers [23]. Thus, extending these contributions is not feasible without re-modeling for the operational semantics of RA [18]. Contributions such as WAtTs [21] and FruitTree [22] extend TMAI with lightweight flow- and 
context-sensitivity. However, they do not capture inter-thread ordering dependencies beyond two threads. Finally, the abstract interpretation technique used in DUET [9] is neither thread-modular nor geared for RA programs. While DUET performs analysis with an unbounded number of threads, it may infer gross overapproximations on some simple programs. Consider the following program where initially $x=0: a: x++\| b: x++$. DUET will infer the value $x=\infty$ at termination. FruitTree [22] also suffers from the same imprecision, though it does not terminate.

Contributions and Outline: In this paper, (C1) as our first contribution, we propose a TMAI technique (see §7) for RA programs using a novel abstract domain which is based on partial orders (PO). The proposed domain succinctly captures abstract ordering dependencies among instructions in a program (see $\S 6)$. While the use of partial orders to analyze concurrency is well-known, to the best of our knowledge this is the first work that formulates the ordering information as an abstract domain. In particular, we model the concrete program semantics as a set of total orders on stores per global variable, also known as modification order (mo)(see §5). A collection of mos are then represented as a PO domain. Notably, the use of PO domain has the following merits: (M1) PO domain is general in its scope and is applicable beyond RA concurrency (see $\S 6.1$ and $\S 6.4$ ). (M2) Introduction of ordering information as a first-class object in abstract interpretation theory permits further abstractions or refinements on the object, an instance of which is presented in contribution $(\mathrm{C} 2)$.

(C2) We present an abstract upper approximation of PO domain (see §6.3) where only the latest stores per thread per variable are preserved and all the older $s b$-ordered stores are forgotten.

(C3) Furthermore, to establish that our analysis preserves soundness and is terminating, we show that (i) the lattice corresponding to the abstract semantics is complete, (ii) establish a Galois connection between the concrete and PO domains, (iii) prove that the abstract upper approximation is sound, and (iv) provide a widening operator to ensure termination of the analysis

(C4) Finally, we implement our proposal in a prototype tool called PRIORI, and demonstrate its effectiveness in refutation and verification of RA programs by comparison with recent state-of-the-art tools in the RA domain (see §8).

We present related work in $\S 2$ followed by an intuitive account of our contributions with the help of examples in $\S 3$.

\section{Related Work}

Weak memory models, in particular C11 model, have been topics of active research in recent years. Many studies have provided proof and logic frameworks $[35,34,24,8]$ and recommended strengthening the C11 models [23, 18]. Many existing contributions have proposed stateless model checking algorithms for RA programs using state-reduction techniques such as dynamic partial order reduction or event structures $[20,2,19,28,25,37]$.

In contrast, there have been relatively fewer investigations of RA concurrency 
using symbolic analysis. While some works have explored using TMAI (which have already been discussed in $\S 1$ ), others have proposed BMC as solutions to verify programs under models such as TSO, PSO and RMO .

Bounded Model Checking. BMC contributions in $[12,1,3]$ operate by placing a bound on the number of loop unrollings or on the number of contexts or both. Dartagnan [12] is a BMC framework that offers support for parameterized reasoning over memory models.While, in principle, Dartagnan can perform bounded reasoning of RA programs, it currently does not support RA semantics.

VBMC [1], a recent BMC solution for RA concurrency, works with an additional bound on the number of views in a RA program - a view of a thread is a collection of timestamps of the latest stores it has observed for each variable. A view-switch takes place when a load operation in a thread, say $t_{2}$, reads from a store in a thread, $t_{1}$, with a timestamp higher than that of any variable in the view of $t_{2}$. While efficient in refutation, VBMC fails to discover property violations in programs which are parametric in the number of readers where the number of view-switches required is beyond the default bound of two (see Appendix A for a detailed discussion).

PO encodings and unfoldings. The use of partial order encodings is diverse and rich in areas of concurrent program verification and testing. The works in $[36,14,11]$ use partial order encodings in dynamic verification tools to predictively reason about multithreaded and message-passing programs. Partial order encoding presented in [3] relies on the axiomatic semantics of memory models such as SC, Intel X86 and IBM POWER and is implemented in a BMC tool. The contributions in [29] and [17] use unfolding semantics to verify and test SC programs, respectively.

A recent study (POET [30]) combines unfolding semantics with abstract interpretation. The solution they have proposed is elegant and close to our proposal, but with several fundamental differences: (D1) POET defines the unfolding under a variant of the independence relation used in the partial order reduction theory [5]. Evidently, the independence relation assumes an interleaving model of computation. While unfoldings can capture true concurrency, the independence relation fundamentally limits their general applicability and restricts POET 's application to only those memory models that can be explained with interleavings. As a result, we have found POET 's technique to be unsound for RA programs. (D2) POET uses unfoldings as an auxiliary object which is external to the abstract interpretation theory. Thus, it is not straightforward to define further abstractions on the unfolding object once created. On the contrary, in our proposal, the PO domain is treated as a first-class object of the abstract interpretation theory, which is open to further abstractions as is witnessed in our contribution (C2). (D3) POET is not thread-modular and navigates an unfolding object of an entire program which is much larger than the PO domains maintained per location per variable in our technique. 


\section{Overview}

We provide an overview of thread-modular analysis using PO domain with the help of small examples.

Let $a$ and $b$ be load and store operations, respectively from different threads to a global memory location. The store $b$ is then called an interference for load $a$ (denoted by $a \rightarrow^{r f} b$, since $b$ can potentially read from $a$ ).

\subsection{Thread Modular Analysis with Partial Order Domain}

Consider the message passing program (MP) shown below on the left. Under RA semantics if $r_{1}=1$, then $r_{2}=0$ is infeasible. Thus, property $P$ is known to be valid.

$$
\begin{aligned}
& \text { (MP) } \\
& \begin{array}{l|l|l}
a: x:=1 & c: r_{1}:=y \\
b: y & :=1 & d: r_{2}:=x
\end{array} \\
& P: r_{1}=1 \Longrightarrow r_{2}=1 \\
& x_{\text {init }}:=0 \quad y_{\text {init }}:=0 \\
& (a \bullet, \square) a: x:=1 \mathrm{rf}^{c}: r_{1}:=y(a \bullet, b \bullet) \\
& \mathrm{sb} \downarrow \quad-\mathrm{Tr}^{-} \downarrow \mathrm{sb} \\
& (a \bullet, b \bullet) b: y:=1^{\mathrm{hb}} d: r_{2}:=x(a \bullet, b \bullet)
\end{aligned}
$$

Program state. Let poset $P O_{x}$ and $V_{x}$ represent the partial order on the observed stores and the abstract value of variable $x \in \mathcal{V}$ where $\mathcal{V}$ is the set of all shared variables in a program. We present the program state (or just state) at each program location (or just location) as a tuple $\left(\Pi_{x \in \mathcal{V}} P O_{x}, \Pi_{x \in \mathcal{V}} V_{x}\right)$, where $\Pi$ is a cartesian product operator defined over indexed family of sets. Consider an execution of (MP) shown above on the right. At location $a$, the state in components is: $P O_{x}=(\{a\}, \emptyset), P O_{y}=(\emptyset, \emptyset), V_{x}=\{1\}, V_{y}=\emptyset$ (Note that the second argument of a poset is the ordering relation). For brevity, we only show the posets of variables (as location-labeled Hasse diagram in a box) and suppress the abstract value in the above and future illustrations.

Interferences. Consider the above MP example again. Thread 1 has no loads; therefore, has no computable interferences. In thread 2, the set of interferences at locations $c$ and $d$ are $\left\{b \rightarrow^{r f} c, \mathrm{ctx} \rightarrow^{r f} c\right\}$ and $\left\{a \rightarrow^{r f} d, \mathrm{ctx} \rightarrow{ }^{r f} d\right\}$, respectively. Note that ctx refers to a special label representing context-i.e., in the absence of any interfering stores, a load instruction will either read from the latest preceding po (program order) store or from the store values that have traveled embedded in the program states up to that load instruction.

TMAI. In the first iteration, the states of thread 1 are computed as shown in the above illustration for locations $a$ and $b$. In thread 2, in the absence of any interefering store, the states are computed with the information from ctx, where $P O_{x}$ and $P O_{y}$ are empty. Therefore, both at $c$ and $d$ we have : $P O_{x}=$ $(\emptyset, \emptyset), P O_{y}=(\emptyset, \emptyset)$.

In the second iteration, the interference $b \rightarrow^{r f} c$ is applied, and the effects of all the instructions prior to $b$ from thread 1 are carried to $c$ and $d$. Thus, at $c$, we have: $P O_{x}=(\{\mathrm{a}\}, \emptyset), P O_{y}=(\{\mathrm{b}\}, \emptyset)$. As a result, the effect of $a$, which is available at $c$ is now also available at $d$ (since it is now part of ctx of thread 2). Thus, the application of interference $a \rightarrow^{r f} d$ becomes redundant. As a matter of fact, the interference $a \rightarrow^{r f} d$ turns out to be infeasible at $d$. This is because 
extending the $P O_{x}$ at $d$ with the $P O_{x}$ at $a$ (by taking the meet of the two orders, see $\S 6.1)$ breaks the acyclicity of $P O_{x}$ at $d$ - one can visualise this by adding an edge from $a$ to itself in the Hasse diagram of the resulting order). In general, to address this issue of invalid application of effects at a state, we introduce the valid extensionality check (see §6.1). Thus, maintaining states this way avoids the need to perform expensive interference infeasibility checks. Notably, such expensive checks are used by other techniques for precision, such as FruitTree [22].

After two iterations, a fix-point is reached. We can now observe that at $d$ there is only a single state reachable when $r 1=1$, which is: $\left(P O_{x}, V_{x}\right)=$ $((\{\mathrm{a}\}, \emptyset), 1),\left(P O_{y}, V_{y}\right)=((\{\mathrm{b}\}, \emptyset), 1)$. Thus the property $P$ is shown to be valid by our analysis.

\subsection{Over-approximating PO Domain}

Posets are history-preserving and their use lends precision to our analysis, however, at the expense of possibly maintaining many posets. We show through a simple example that with further abstraction of forgetting older sb-ordered stores in the posets (see C2) one can obtain succinct posets, thereby resulting in fewer abstract states, in many scenarios. Consider the two example posets (leftmost and center) on vari$a \quad a \bullet \quad$ able $x$ denoting two distinct states at a location in a $d \quad \bigvee^{d} d$ program as shown in Figure 2. Assume that stores $a$ $b \bullet \quad b \bullet \quad$ and $b$ are $s b$-ordered and store $d$ is from a different

Fig. 2: Two posets and an abstract poset thread. By forgetting the older $s b$-ordered store $a$, a smaller abstract $P O_{x}$ is obtained, which is shown as the rightmost poset in the figure. Notice that for two distinct states with differing posets at a location, the same abstract poset is obtained; consequently a single abstract state. This results in a smaller abstract state graph. However, if the value of store at $a$ was read in a variable that affected an assertion, then the over-approximated abstract state could result in a loss of precision leading to a possible false positive. A detailed example program corresponding the illustrated example posets can be found in Appendix B.

\section{Preliminaries}

RA semantics. Given a multithreaded program $P:=\|_{i \in \text { Tid }} P_{i}$, where Tid $=$ $\{1, \ldots, n\}$ is the set of thread ids and $\|$ is a parallel composition operator. Let $\mathcal{V}$, and $\mathcal{L}$ be the set of shared variables and set of program locations, respectively. We use $(\ell, i)$ to denote the event corresponding to the $i^{\text {th }}$ occurrence of program instruction labeled $\ell$. Let St, Ld and RMW be the set of all store, load and rmw (read-modify-write) events from $P$, respectively. We denote relations sequencedbefore and reads-from of RA model $[24,4]$ by $\rightarrow^{s b}$ and $\rightarrow^{r f}$, respectively . The notation $a \rightarrow^{s b} b$ and $s \rightarrow^{r f} l$ represents $(a, b) \in \rightarrow^{s b}$ and $(s, l) \in \rightarrow^{r f}$, respectively. The happens-before ( $\mathrm{hb})$ relation for RA concurrency is defined as a transitive 
closure $\left(\rightarrow^{r f} \cup \rightarrow^{s b}\right)^{+}$. Let $\left(M_{x}, \leqslant M_{x}\right)$ denote the modification order (mo) over a set of store and rmw events $M_{x} \subseteq$ St $\cup$ RMW to a memory location $x$ in a program execution. As defined in [24,4], every valid RA program execution must have a mo that is consistent with hb.

Loset. The total ordering relation $\leqslant_{M_{x}}$ is a relation between every pair of stores $w_{1}, w_{2} \in M_{x}$ in a program execution (alternatively represented as $w_{1} \leqslant M_{x} w_{2}$ ). We alternatively refer to a modification order as a loset (linearly ordered set). Let $M^{S}$ be the the set of all possible linear orderings over the set $S \subseteq$ St $\cup$ RMW. Let $L(S, \preccurlyeq)$ be a function that gives all possible linearizations of elements in $S \subseteq$ St $\cup$ RMW that respect the set of ordering constraints $\preccurlyeq$ (note the difference with $\leqslant)$. For example $L(\{a, b\}, \emptyset)$ will result in $\{\{(a, b)\},\{(b, a)\}\}$. Similarly, $L(\{a, b, c\},\{(a, b),(a, c)\})$ will produce: $\{(a, b),(a, c),(b, c)\}$ and $\{(a, b),(a, c)$, $(c, b)\}$.

Interference. Following the description of interferences in $\S 3$, we define interference as a relation $\mathcal{I} \subseteq \mathrm{Tid} \times \mathrm{Ld} \times(\mathrm{St} \cup \mathrm{RMW})$ such that $\mathcal{I}(t)(\mathrm{ld}) \stackrel{\text { def }}{=} \operatorname{ctx} \cup \mathrm{St} \cup \mathrm{RMW}$, ctx is the store in the program state at some label in pre(ld) for thread $t$. We define $\operatorname{pre}(\mathrm{ld})$ as the set of labels immediately preceding $1 \mathrm{~d}$ in $s b$ order.

\section{Concrete Semantics}

We consider the set of mo losets per global variable as concrete semantics of a program. Evidently, the set of mo losets is already a sound over-approximation of the set of concrete executions (see Defn. 5 in [24]). Thus, considering the set of mo losets as concrete program semantics does not break the soundness of our analysis framework [7]; in fact, it serves the purpose of keeping the concrete semantics expressible enough while maintaining the ease of further abstractions.

\subsection{Modification Orders as Posets}

We define the concrete/collecting semantics by the set $\mathcal{T}$ such that each element $t \in \mathcal{T}$ is a subset of $M^{S}$ where $S \subseteq$ St $\cup$ RMW. Let $t_{1} \stackrel{\text { def }}{=}\left(S_{1},\{\leqslant\}_{S_{1}}\right)$ and $t_{2} \stackrel{\text { def }}{=}\left(S_{2},\left\{\leqslant \mathbb{\}}_{S_{2}}\right)\right.$ be two elements of $\mathcal{T}$, where $\{\leqslant \leqslant\}_{S}$ denotes a set of losets over $S$ i.e. $\{\leqslant \leqslant\}_{S}=\left\{\leqslant_{1}, \leqslant_{2}, \ldots\right\}$. Two elements $t_{1}, t_{2} \in \mathcal{T}$ are related by an ordering relation $\subseteq$, denoted by $t_{1} \subseteq t_{2}$. The definition of the ordering relation is as follows.

Definition $1 t_{1} \subseteq t_{2} \Longleftrightarrow\left(S_{1} \supseteq S_{2} \wedge \forall \leqslant \leqslant_{i} \in\left\{\leqslant \mathbb{\}}_{S_{1}} \exists \leqslant j \in\{\leqslant\}_{S_{2}} . \forall a, b \in\right.\right.$ $\left.S_{2} a \leqslant_{i} b \Longrightarrow a \leqslant \leqslant_{j} b\right)$.

We extend the set $\mathcal{T}$ with a special element $\perp_{\mathcal{T}}$ such that $\forall t \in \mathcal{T} . \perp_{\mathcal{T}} \subseteq$ $t$. Each element in $\mathcal{T}$ is a set of mo losets that represents a set of (possibly partial) executions. For instance, $t_{1}$ in Figure $3 \mathrm{a}$ is an over-approximation of all the executions whose mos satisfy either $m_{11}$ or $m_{12}$. Note $t_{1} \subseteq t_{2}$, which means that the set of executions corresponding to $t_{2}$ is larger than the set of executions corresponding to $t_{1}$. We infer that $t_{1}$ gives us more precise information 


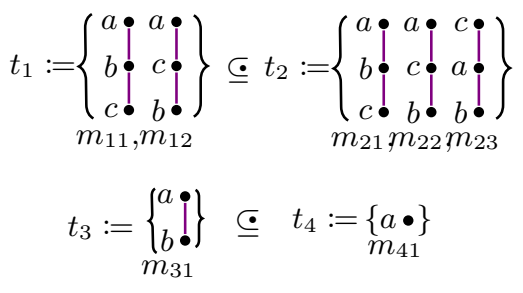

(a)

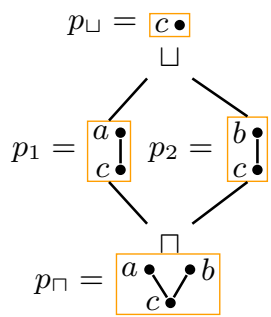

(b)

Fig. 3: Orderings over $\mathcal{T}, \mathcal{P}$

on execution possibilities than $t_{2}$ for the same program. Similarly, in Figure 3a element $t_{3}$ is ordered below $t_{4}$. The set of executions having $m_{41}$ as a part of their mo is larger than set of executions having $m_{31}$ as part of their mo.

The element $\perp_{\mathcal{T}}$ represents a set in which all modification orders are inconsistent, and hence represents an invalid execution. Likewise, we introduce element $\top_{\mathcal{T}}=(\emptyset, \emptyset)$ in the $\mathcal{T}$ representing an empty set of constraints, which is equivalent to the set of all valid executions. By definition, $\top_{\mathcal{T}}$ is ordered above all the elements $\mathcal{T}$ in the $\subseteq$. We establish that $\mathcal{T}$ is a poset under the relation $\subseteq$.

Lemma 1. $(\mathcal{T}, \subseteq)$, is a poset. ${ }^{1}$

\section{$6 \quad$ Abstract Semantics}

We present a two-layered abstraction to arrive at final abstract RA program semantics. In particular, (i) the set of mo losets of a program is abstracted in to PO domains, and (ii) the PO domains are further over-approximated, where for each variable all stores older than the latest store under sb ordering in its poset are forgotten. Further, we demonstrate that abstract semantics produced in step (i) from above forms a complete lattice and establish a Galois connection between the concrete and abstract domains.

\section{1 mo Posets as Lattices}

In this section we define a lattice over $\mathcal{P}$ which is the set of all partial orders. We use the terms mo poset and PO domain interchangeably for this lattice.

We combine two or more mo losets and respresent them as a collection of partial orders. For instance, consider mo losets $p_{1}$ and $p_{2}$ (shown in Figure $3 \mathrm{~b}$ ) in $\mathcal{P}$. These can be combined in the following two ways: (i) the orderings in $p_{1}$ and $p_{2}$ are both present in the combination (the binary operator is denoted by $\sqcap$ and the resulting element is denoted by $p_{\sqcap}$ ), or (ii) common orderings in $p_{1}$ and $p_{2}$

\footnotetext{
${ }^{1}$ Proofs of all lemmas and theorems in this article are available in the Appendix C
} 
on the common elements are present in the combination (the binary operator is denoted by $\sqcup$ and the resulting element is denoted by $\left.p_{\sqcup}\right)$. After the application of step (i), we note that the pairs $(a, b)$ or $(b, a)$ are not in the relation $p_{1} \sqcap p_{2}$. Similarly, after the application of step (ii), we note that all those executions that contain $c$ are included in $p_{\sqcup}$. Also, note that $p_{\sqcap}, p_{\sqcup} \in \mathcal{P}$. Going forward we define the following operations over the elements in a set of partial orders:

Less $\left(p_{1} \sqsubseteq p_{2}\right)$ : An ordering relation among two partial orders $\left.p_{1}=\left(M_{x}, \preccurlyeq{ }_{1}\right)\right)$ and $\left.p_{2}=\left(N_{x}, \preccurlyeq 2\right)\right), p_{1}, p_{2} \neq \perp$ is defined as following: $p_{1} \sqsubseteq p_{2} \Longleftrightarrow M_{x} \supseteq$ $\left.N_{x} \wedge a \preccurlyeq 2 b \Longrightarrow a \preccurlyeq r_{1} b\right)$ and $\forall p \in \mathcal{P}, \perp \sqsubseteq p$

Is Consistent $\left(p_{1} \uparrow p_{2}\right)$ : Two partial orders are consistent with each other if they do not contain any conflicting pair and $\perp$ is not consistent with any element. Formally, $\perp \uparrow p_{2} \stackrel{\text { def }}{=}$ false, $p_{1} \uparrow \perp \stackrel{\text { def }}{=}$ false and $\forall p_{1}, p_{2} \neq \perp$,

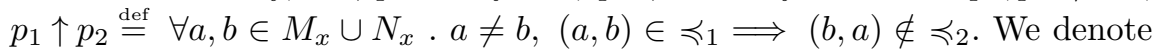
inconsistent partial orders using the notation $p_{1} \uparrow p_{2}$.

Is Valid Extension $(p \triangleleft \mathbf{s t})$ : A store event st is a valid extension of the partial order $p=\left(M_{x}, \preccurlyeq\right)$ iff there is no instruction ordered after st in the ordering relation $\preccurlyeq$. Formally, $p \triangleleft$ st $\stackrel{\text { def }}{=} \forall a \in M_{x},(s t, a) \notin \preccurlyeq$. A invalid extension of a partial order $p$ by a store $s t$ is denoted by $p \nless \mathrm{st})$.

Append $(p \diamond s t):$ Appends the store operation st at the end of modification order $p=\left(M_{x}, \preccurlyeq\right)$ if st is a valid extension of $p$ i.e. $p \diamond$ st $\stackrel{\text { def }}{=}$ if $p \triangleleft$ st then $\left(M_{x} \cup\{\mathrm{st}\}, \preccurlyeq \cup\left\{(a, s t) \mid a \in M_{x}\right\}\right)$ else $\perp$.

Meet $\left(p_{1} \sqcap p_{2}\right)$ : The meet of two partial orders $\left.p_{1}=\left(M_{x}, \preccurlyeq 1\right)\right)$ and $p_{2}=$ $\left.\left(N_{x}, \preccurlyeq_{2}\right)\right)$ is defined as: $p_{1} \sqcap p_{2} \stackrel{\text { def }}{=}$ if $p_{1} \uparrow p_{2}$ then $\left(M_{x} \cup N_{x}, \preccurlyeq 1 \cup \preccurlyeq 2\right)$ else $\perp$.

Join $\left(p_{1} \sqcup p_{2}\right)$ : The join of two partial order $\left.p_{1}=\left(M_{x}, \preccurlyeq_{1}\right)\right)$ and $p_{2}=\left(N_{x}, \preccurlyeq 2\right)$, $p_{1}, p_{2} \neq \perp$ is defined as the intersection of common ordered pairs in the partial orders, i.e, $p_{1} \sqcup p_{2} \stackrel{\text { def }}{=}\left(M_{x} \cap N_{x}, \preccurlyeq 1 \cap \preccurlyeq 2\right)$. We define $\perp \sqcup p_{2} \stackrel{\text { def }}{=} p_{2}$ and $p_{1} \sqcup \perp \stackrel{\text { def }}{=} p_{1}$.

Widening $\left(p_{1} \nabla p_{2}\right)$ : The widening operator over two partial orders $p_{1}=\left(M_{x}\right.$, $\preccurlyeq 1)$ and $p_{2}=\left(N_{x}, \preccurlyeq 2\right), p_{1}, p_{2} \neq \perp$ is defined as $p_{1} \nabla p_{2} \stackrel{\text { def }}{=}\left(Q_{x}, \preccurlyeq\right)$, where $Q_{x}=\left\{a \mid a=(\ell, i) \in M_{x} \cap N_{x} \wedge \nexists b=(\ell, j) \in M_{x} \cap N_{x} \cdot j<i\right\}$ and $\preccurlyeq=\left\{(a, b) \mid(a, b) \in \preccurlyeq 1 \cap \preccurlyeq 2 \wedge a, b \in Q_{x}\right\}$. We define $\perp \nabla p_{2} \stackrel{\text { def }}{=} p_{2}$ and $p_{1} \nabla \perp \stackrel{\text { def }}{=} p_{1}$.

Lemma 2. The operators $\sqcup$ and $\sqcap$ define the lub and glb of any two elements of $\mathcal{P}$, respectively. ${ }^{1}$

Lemma 3. ( $\mathcal{P}, \sqsubseteq, \sqcup, \sqcap, \perp, \top)$ is a complete lattice, where $\mathcal{P}$ is set of all possible partial orders over elements of set $\mathrm{St} \cup \mathrm{RMW}, \top$ is defined as empty poset, and $\perp$ is a special element that is ordered below all the elements of $\mathcal{P}$ in $\sqsubseteq .1$

The proof of Lemma 3 follows from Lemma 2, the definition of $\sqcup$ and $\sqcap$ operations of $\mathcal{P}$, and standard properties of operators.

Lemma 4. The binary operation $\nabla$ defines a widening operator over the elements of the lattice $(\mathcal{P}, \sqsubseteq, \sqcup, \sqcap, \perp, \top) .{ }^{1}$ 
We explain the widening operator $\nabla$ using an example. Recall that each element of lattice $\mathcal{P}$ is a partial order over program events. Let $p=\left(Q_{x}, \preccurlyeq\right)=$ $p_{1} \nabla p_{2}$, then the set of events in $p$ maintains the earliest occurrence of common events in $M_{x}$ and $N_{x}$ corresponding to $p_{1}$ and $p_{2}$, respectively. Consider the events $e_{2}=(\ell, 2)$ and $e_{3}=(\ell, 3)$, which are generated by the same program instruction labeled $\ell$. If both $p_{1}$ and $p_{2}$ contain the ordering $e_{2}$ and $e_{3}$, then the result of widening will contain the earliest occurrence of an event from $\ell$, i.e., $e_{2}$ so long as $e_{1}=(\ell, 1) \notin M_{x} \cap N_{x}$. The set of orderings $\preccurlyeq$ is defined over the elements of $Q_{x}$. Hence no ordering involving $e_{3}$ in this example will be in $\preccurlyeq$.

Given a monotone function $f: \mathcal{P} \rightarrow \mathcal{P}$, consider the chain $f_{\nabla}^{0}, f_{\nabla}^{1}, f_{\nabla}^{2} \ldots$ with $f_{\nabla}^{0}=\perp$ and $f_{\nabla}^{i}=f_{\nabla}^{i-1} \nabla f\left(f_{\nabla}^{i-1}\right)$ for some $i>0$. An essential requirement on $\nabla$ for it to be a widening operator is that the above chain must stabilize, i.e., $f\left(f_{\nabla}^{n}\right) \sqsubseteq f_{\nabla}^{n}$ for some $n>0$. It means that the function $f$ is reductive at $f_{\nabla}^{n}$. We show in the proof of Lemma 4 that our defined operator $\nabla$ is indeed a widening operator. Using Tarski's fixpoint theorem, it follows that $\operatorname{lfp}(f) \sqsubseteq f_{\nabla}^{n}$, where $\operatorname{lfp}(f)$ is the least fixed point of $f$. As a result, $f_{\nabla}^{n}$ is a sound over-approximation of $f$, which guarantees termination of analysis with infinite lattices having infinite ascending chains.

Definition 2 The abstraction function $\alpha: \mathcal{T} \rightarrow \mathcal{P}$ is defined as $\alpha\left(\perp_{\mathcal{T}}\right) \stackrel{\text { def }}{=} \perp$ and $\forall t \neq \perp_{\mathcal{T}}, \alpha(t) \stackrel{\text { def }}{=}\left(M_{x}, \preccurlyeq\right)$ for some $t=(S,\{\leqslant\})$ given $M_{x}=S$, and $\preccurlyeq=\bigcap \leqslant{ }_{i}$.

Definition 3 The concretization function $\gamma: \mathcal{P} \rightarrow \mathcal{T}$ is defined as $\gamma(\perp) \stackrel{\text { def }}{=} \perp_{\mathcal{T}}$ and $\forall p \neq \perp, \gamma(p) \stackrel{\text { def }}{=}(S,\{\leqslant\})$ for some $p=\left(M_{x}, \preccurlyeq\right)$ given $S=M_{x}$ and $\{\leqslant\}$ is set of all possible linearizations of $\preccurlyeq$ i.e. $\{\leqslant\}\}=L(S, \preccurlyeq)$.

Having defined the abstraction and concretization operators, we can now establish the Galois connection between the poset $\mathcal{T}$ and the lattice $\mathcal{P}$.

Theorem 1. $(\mathcal{T}, \subseteq) \underset{\alpha}{\stackrel{\gamma}{\leftrightarrows}}(\mathcal{P}, \sqsubseteq, \sqcup, \sqcap, \perp, \top) .{ }^{1}$

We lift the result from Theorem 1 to the product lattices of all the program variables. Theorem 2 articulates that the Galois connection between concrete and abstract product lattices is preserved.

Theorem 2. The correspondence between $\prod_{x \in \mathcal{V}} \mathcal{P}_{x}$ and $\prod_{x \in \mathcal{V}} \mathcal{T}_{x}$ is a Galois connection. ${ }^{1}$

It is worthwhile to note that lattice $\mathcal{P}$ is not tied to the RA semantics. As such, the $\mathrm{PO}$ domain is not specific to any memory model. We present a discussion in $\S 6.4$, on the applicability of PO domain beyond RA semantics. Below, we give a description of transfer functions for the operations in RA programs.

\subsection{Abstract Semantics of RA programs}

The values of shared variables in the program can be abstracted to any known numeric abstract domain such as interval, octagon, or polyhedra. Let $\mathbb{V}^{\sharp}$ represents the set of values in the chosen abstract domain. Let $\mathcal{M}: \mathcal{V} \rightarrow \mathbb{V}^{\sharp}$ define 


$$
\begin{aligned}
& (\operatorname{pre}(\ell), m o, m) \in \mathcal{S} \quad m^{\prime}=m[x \rightarrow v] \\
& \begin{array}{l}
m o^{\prime}=m o[x \rightarrow m o(x) \diamond \ell] \\
\mathcal{S} \stackrel{\ell: \text { st } x v}{\longrightarrow} \mathcal{S} \uplus\left(\ell, m o^{\prime}, m^{\prime}\right)
\end{array} \text { STORE } \\
& (\operatorname{pre}(\ell), m o, m) \in \mathcal{S} \\
& (\operatorname{pre}(\ell), m o, m) \stackrel{\ell: 1 \mathrm{~d} x}{\longrightarrow}\left(\ell, m o^{\prime \prime}, m^{\prime \prime}\right) \\
& m^{\prime \prime}(x)=v \\
& \frac{\left(\operatorname{pre}(\ell), m o^{\prime \prime}, m^{\prime \prime}\right) \stackrel{\ell: \text { st } x v^{\prime}}{\longrightarrow}\left(\ell, m o^{\prime}, m^{\prime}\right)}{\mathcal{S} \stackrel{\ell: \text { rmw } x v v^{\prime}}{\longrightarrow} \mathcal{S} \uplus\left(\ell, m o^{\prime}, m^{\prime}\right)} \mathrm{RMW} \\
& \left(\operatorname{pre}(\ell), m o_{l}, m_{l}\right) \in \mathcal{S} \quad \text { st } \in \mathcal{I}(t)(\ell) \\
& \left(\text { st, } m o_{s}, m_{s}\right) \in \mathcal{S} \\
& \left(\operatorname{pre}(\ell), m o^{\prime}, m^{\prime \prime}\right)=\mathrm{AI}\left(\left(\operatorname{pre}(\ell), m o_{l}, m_{l}\right),\left(\text { st }, m_{s}, m_{s}\right)\right) \\
& m^{\prime}=m^{\prime \prime}\left[x \rightarrow m_{s}(x)\right] \\
& \mathcal{S} \stackrel{\ell: 1 \mathrm{~d} x}{\longrightarrow} \mathcal{S} \uplus\left(\ell, m o^{\prime}, m^{\prime}\right)
\end{aligned}
$$

Fig. 4: Transfer functions for RA programs. $\mathrm{AI}\left(\left(\ell_{1}, m o_{1}, m o_{2}\right),\left(\ell_{2}, m_{2}, m_{2}\right)\right) \stackrel{\text { def }}{=}$ $\left(\ell_{1},\left(m o_{1} \diamond \ell_{2}\right) \sqcap m o_{2}, m_{1} \sqcup m_{2}\right)$; AI applies the interference from $\left(\ell_{2}, m o_{2}, m_{2}\right)$ to the memory and mo poset state of $\left(\ell_{1}, m o_{1}, m o_{2}\right)$.

the memory state of a program. Let $\mathbb{M}: \mathcal{V} \rightarrow \mathcal{P}$ represent a map from shared variables to corresponding elements in the abstract mo poset lattice $\mathcal{P}$. We abuse notations $\diamond, \sqcup, \sqcap, \nabla, \uparrow$, and $\triangleleft$ to represent the corresponding pointwise-lifted operators for M. For instance, the pointwise lifting of $\diamond$ appends the stores of variable $v$ only to its modification order $(i . e ., \mathbb{M}(v))$; the modification orders $\mathbb{M}\left(v^{\prime}\right)$ for variables $v^{\prime} \neq v$ remain unchanged. The pointwise lifting for other operators is straighforward. From Theorem 2, it follows that M along with the pointwise lifted operators constitute the sought abstract domain.

Let $\Sigma \subseteq \mathcal{L} \times(\mathbb{M} \times \mathcal{M})$ represents the set of all reachable program states. The transfer functions for operations ld, st and rmw are defined in Figure 4. We provide additional rules (for lock and unlock) and auxillary functions, which are supported by our technique, in Appendix D. Since we assume the SSA representation of programs, arithmetic operations only modify the thread local variables. As a result, $\mathbb{M}$ remains unchanged. The effects of arithmetic operations on shared variables is captured via numeric abstract domains. Thus, the transfer functions for such operations are excluded from our presentation. The semantic definitions in Figure 4 are parameterized in terms of the set of currently explored reachable program states, $\mathcal{S} \subseteq \Sigma$, at a some point during the analysis.

Consider the LOAD rule which, defines the semantics of a load operation. A load of a shared variable $x$ at $\ell$ is performed at program state(s) $\mathcal{S}$ using the following steps. Let st be an interfering instruction for $\ell$. Each explored program state $\left(\mathrm{st}, \mathrm{mo}_{s}, m_{s}\right)$ at instruction label st is considered as an interference and analyzed with the set of program states at label pre $(\ell)$ using the function AI (defined in the caption of Figure 4). When the interference from program 
state $\left(\ell_{2}, m_{2}, m_{2}\right)$ is successfully applied to the program state $\left(\ell_{1}, m o_{1}, m o_{2}\right)$ by function AI (the load at $\ell_{1}$ reads from the store at $\ell_{2}$ ), then as a result $\ell_{2}$ is appended in the partial order at $\ell_{1}$, i.e., $m o_{1}$. For all other events prior to $\ell_{1}$ and $\ell_{2}$, the precise ordering information among them is computed by taking the meet of $m o_{1}$ and $m o_{2}$, i.e., $m o_{1} \sqcap m o_{2}$ (because the ordering of such events must be consistent with both $m o_{1}$ and $m o_{2}$ ).

In the state at $\ell_{1}$, the value of variables other than interfering variable $x$ can come from either $m_{1}$ or $m_{2}$. The function AI joins the maps $m_{1}$ and $m_{2}$ to obtain all feasible values for such variables. To compute $\sqcup$ on memory values, one can choose abstract domains such as intervals or octagons. Let AI return ( $\left.p r e(\ell), m o^{\prime}, m^{\prime \prime}\right)$ when the interference is applied from $\left(\mathrm{st}, \mathrm{mo}_{s}, m_{s}\right)$ to $\left(\operatorname{pre}(\ell), m_{l}, m_{l}\right)$. The value of variable $x$ read by the load operation $\ell$ in the program state $\left(\operatorname{pre}(\ell), m o^{\prime}, m^{\prime \prime}\right)$ will be the same as the value of variable $x$ in the interfering program state $m_{s}(x)$. Thus, we substitute $m^{\prime \prime}(x)$ with $m_{s}(x)$ to construct the reachable program state $\left(\ell, m o^{\prime}, m^{\prime}\right)$.

Finally, the resulting state at $\ell$ is combined with the currently existing states by the $\uplus$ operator. The operator $\uplus$ performs instruction-wise join of states, i.e., it joins the memory state of two states if their instruction labels and mo posets are the same. It also joins the mo poses if the instruction label and the memory states are the same, otherwise, it leaves the two states as is. Formally, the operation $\uplus$ replaces any two program states (say $\left(\ell_{1}, m o_{1}, m_{1}\right)$ and $\left.\left(\ell_{2}, m_{2}, m_{2}\right)\right)$, with a single program state $\left(\ell_{1}, m o_{1}, m\right)$, where $m=m_{1} \sqcup m_{2}$, if $m o_{1}=m o_{2} \wedge \ell_{1}=\ell_{2}$, and with $\left(\ell_{1}, m o, m_{1}\right)$, where $m o=m o_{1} \sqcup m o_{2}$, if $m_{1}=m_{2} \wedge \ell_{1}=\ell_{2}$.

Transfer functions for RMW and STORE can be interpreted in a similar way. Readers may note that, in general, two successful RMW operations will never read from the same store as is assumed in our rule. However, our definition is sound (and simple to understand); we provide a more precise definition in $\S 7.2$, which is also implemented in PRIORI.

\subsection{Abstracting the Abstraction: Approximating mo Posets}

We leverage the ordering rules of the RA memory model to further abstract the modification orders. Let $p \stackrel{\text { def }}{=}\left(Q_{x}, \preccurlyeq\right), p_{1} \stackrel{\text { def }}{=}\left(M_{x}, \preccurlyeq 1\right), p_{2} \stackrel{\text { def }}{=}\left(N_{x}, \preccurlyeq 2\right), p_{a} \stackrel{\text { def }}{=}$ $\left(A_{x}, \preccurlyeq_{a}\right)$ be some elements in $\mathcal{P}$. We shall use these definitions whenever $p, p_{1}, p_{2}$ and $p_{a}$ appear in definitions and predicates below.

Our abstraction function $\alpha^{\sharp}: \mathcal{P} \rightarrow \mathcal{P}$ can be defined as follows: $\alpha^{\sharp}(\perp)=\perp$ and $\forall p \neq \perp, \alpha^{\sharp}(p) \stackrel{\text { def }}{=}\left(A_{x}, \preccurlyeq a\right)$, where $A_{x}=Q_{x} \backslash\left\{a \mid \exists b \in Q_{x} . a \rightarrow \rightarrow^{s b} b \wedge a \neq b\right\}$ and $\preccurlyeq a_{a}=\preccurlyeq \backslash\left\{(a, b) \mid(a, b) \in \preccurlyeq \wedge\left(a \notin A_{x} \vee b \notin A_{x}\right)\right\}$.

Soundness of $\alpha^{\sharp}$ Abstraction: Let relation $\beta \in \wp(\mathcal{P} \times \mathcal{P})$, where $\wp$ denotes power set, be defined as $\exists p_{1}, p_{2} \in \mathcal{P},\left(p_{1}, p_{2}\right) \in \beta \Longleftrightarrow p_{1}=\perp \vee\left(N_{x} \subseteq M_{x} \backslash\{a \mid\right.$ $\left.\left.\exists b \in M_{x} . a \rightarrow^{s b} b \wedge a \neq b\right\} \wedge \preccurlyeq_{2} \subseteq \preccurlyeq_{1}\right)$. Through Lemma 5 we establish that our definition of $\beta$ indeed provides a soundness relation.

Lemma 5. $\left(p_{1}, p_{2}\right) \in \beta \Longrightarrow p_{1} \sqsubseteq p_{2}{ }^{1}$

Lemma 6. Abstract soundness assumption holds under $\beta$, i.e., $\forall p, p_{1}, p_{2} \in \mathcal{P}$. $\left(p, p_{1}\right) \in \beta \wedge p_{1} \sqsubseteq p_{2} \Longrightarrow\left(p, p_{2}\right) \in \beta .{ }^{1}$ 
In other words, Lemma 6 allows us to conclude that if $p_{1}$ is a sound overapproximation of $p$, then every element ordered above $p_{1}$ in lattice $\mathcal{P}$ is also a sound over-approximation of $p$ under $\beta$. We shall use Lemmas 5-6 to establish the soundness of $\alpha^{\sharp}$ in the theorem below.

Theorem 3. Abstraction relation $\alpha^{\sharp}$ is minimal sound abstraction under soundness relation $\beta$, i.e., $\left(p_{1}, p_{2}\right) \in \beta \Longleftrightarrow \alpha^{\sharp}\left(p_{1}\right) \sqsubseteq p_{2} .{ }^{1}$

The proof of Theorem 3 is obtained by a straightforward application of the definitions of $\alpha^{\sharp}, \beta$ and Lemma 6.

We redefine some of the operations described in $\S 6.1$ in order to assist with the computation of transfer functions under the $\alpha^{\sharp}$ abstraction:

Is Consistent $\left(p_{1} \uparrow p_{2}\right): \perp \uparrow p_{2} \stackrel{\text { def }}{=}$ false, $p_{1} \uparrow \perp \stackrel{\text { def }}{=}$ false and $\forall p_{1}, p_{2} \neq \perp$ $p_{1} \uparrow p_{2} \stackrel{\text { def }}{=} \forall a, b\left((a, b) \in \preccurlyeq 1 \Longrightarrow \forall b \rightarrow{ }^{s b} c .(c, a) \notin \preccurlyeq 2\right) \wedge\left((a, b) \in \preccurlyeq_{2} \Longrightarrow\right.$ $\left.\forall b \rightarrow{ }^{s b} c .(c, a) \notin \preccurlyeq 1\right)$. Note that $\rightarrow^{s b}$ is reflexive. As before, we use the notation $p_{1} \uparrow p_{2}$ when $p_{1}$ and $p_{2}$ are inconsistent.

Is Valid Extension $(p \triangleleft \mathbf{s t}): p \triangleleft$ st $\stackrel{\text { def }}{=} \forall a(s t, a) \notin \preccurlyeq \wedge \nexists b \in Q_{x} . s t \rightarrow^{s b} b$. We use the notation $p \Varangle \mathbf{s t}$ to indicate that st is not a valid extension of $p$.

Append ( $p \diamond \mathbf{s t})$ : If $\mathbf{s t}$ is a valid extension of $p$, then append the store operation st at the end of partial order $p$ and delete the older instructions, if any, i.e. $p \diamond \mathrm{st} \stackrel{\text { def }}{=}$ if $p \triangleleft$ st then $\left(Q_{x} \cup \mathrm{st} \backslash\left\{a \mid a \rightarrow^{s b}\right.\right.$ st $\}, \preccurlyeq \cup\{(a, s t) \mid a \in$ $\left.Q_{x}\right\} \backslash\left\{(a, b) \mid\left(a \rightarrow^{s b}\right.\right.$ st $\wedge a \neq$ st $) \vee\left(b \rightarrow{ }^{s b}\right.$ st $\wedge b \neq$ st $\left.\left.)\right\}\right)$ else $\perp$.

\section{Over-Approximating the Semantics of RA programs.}

We use the modified definitions of $\diamond, \uparrow, \uparrow, \triangleleft$ and $\Varangle$ operators to perform analysis under $\alpha^{\sharp}$ abstraction. The semantics of st, ld and rmw operations and the set of all program states $\Sigma$ remain the same as under $\alpha^{\sharp}$, as defined in $§ 6.2$.

\subsection{Posets as a Generic Abstraction}

In this section, we discuss the possibility of using the lattice $(\mathcal{P}, \sqsubseteq, \sqcup, \sqcap, \perp, \top)$ as a generic abstraction, and using it for reasoning memory models other than RA. As a first step, we reinvestigate how we define the collecting semantics for programs under non-RA memory models. The mo losets may not be best suited collecting semantics to reason over programs under other memory models.

Consider, for instance, the TSO model. The collecting semantics for TSO model require an ordering over all the events of shared variables in the program, except among the store-load pairs of different variables from the same thread. Thus, using losets as concrete semantics over loads and stores of all the shared variables in which the store-load pair of different variables in a thread can appear in any order will suffice. This allows us to capture $\mathrm{rfe}$ (reads-from-external, $\mathrm{rfe}=\mathrm{rf} \backslash \mathrm{po}$ ) in the loset. Similarly, considering the PSO model the concrete semantics containing one loset per variable containing all the load and store events of that variable will suffice.

Note that once the collecting semantics is suitably fixed, then formal objects such as $(\mathcal{T}, \subseteq),(\mathcal{P}, \sqsubseteq, \sqcup, \sqcap, \perp, \top)$, and functions $\alpha$ and $\gamma$ can be used in the 


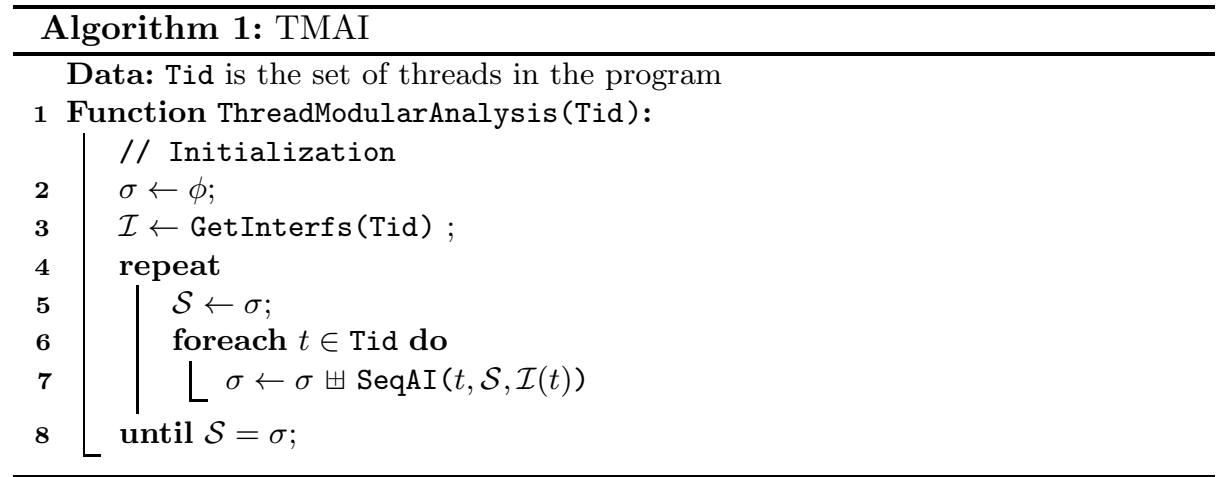

analysis without requiring any change. However, designing $\alpha^{\sharp}$ for other memory models may require careful analysis, and is left as future work.

\section{Thread-modular Abstract Interpretation}

\subsection{Analysis Algorithms}

We present Algorithm 1 in which procedure ThreadModularAnalysis analyzes the entire program by considering one thread at a time. The analysis begins with the initialization of the set of explored program states (line 2). For each thread $t \in$ Tid, relation $\mathcal{I}(t)$ is computed (line 3 ) according to the definition in $\S 4$. Each thread is analyzed under all possible interferences in $\mathcal{I}$ until a fixed point is reached (lines 4-8). The function $\operatorname{SeqAI}(\mathrm{t}, \mathcal{S}, \mathcal{I}(\mathrm{t}))$ is a standard work-list based sequential abstract interpretation over a single thread [27]. Our work adapts this analysis by replacing the transfer functions with the ones given in $\S 6.2$. The function returns a set of states for all the locations in the thread $t$. The operator $\uplus$ performs instruction-wise join (explained in §6.2) of environments in the existing $(\sigma$, line 5$)$ and the newly computed program states $(\operatorname{SeqAI}(\mathrm{t}, \mathcal{S}, \mathcal{I}(\mathrm{t})))$. The details of RA memory model, interferences, abstractions and semantics of transfer functions are all embedded in line 7 of the algorithm.

\subsection{A Note on Precision}

When the older $s b$-ordered stores are forgotten in a mo poset and those program states having the same mo poset are combined, it results in the merging of multiple program executions into a single over-approximation. In theory, it is possible that one or more forgotten (older) stores were critical to prove the property. We can achieve higher precision if we can discern such critical stores and preserve the ordering constraints over such stores in the mo posets.

In our study, we found that many benchmarks that model mutual exclusion under the RA memory model use rmw instructions as synchronization fences. 
These rmw events are instances of critical stores, and we flag them as such and preserve all the older rmw instructions in $\preccurlyeq x$.

Updated Semantics of RMW The semantics of RMW given in Figure 4 (for a shared variable $x$ ), while sound, are not precise according to RA semantics. We update the semantics in the following way: the consistency check of two elements $p_{1}=\left(M_{x}, \preccurlyeq M_{x}\right)$ and $p_{2}=\left(N_{x}, \preccurlyeq N_{x}\right)$ will return true iff $p_{1} \uparrow p_{2} \wedge \forall \mathrm{rmw}_{1} \in$ $p_{1}, \mathrm{rmw}_{2} \in p_{2},\left(\left(\mathrm{rmw}_{1}, \mathrm{rmw}_{2}\right) \in \preccurlyeq M_{x} \vee\left(\mathrm{rmw}_{1}, \mathrm{rmw}_{2}\right) \in \preccurlyeq N_{x} \vee\left(\mathrm{rmw}_{2}, \mathrm{rmw}_{1}\right) \in \preccurlyeq M_{x}\right.$ $\left.\vee\left(\mathrm{rmw}_{2}, \mathrm{rmw}_{1}\right) \in \preccurlyeq N_{x}\right)$. The mentioned update prohibits the combination of those two partial orders such that if they were to be combined then the rmw events no longer remain in a total order.

\subsection{Loops and Termination}

Widening [6] is generally used to handle non-terminating loops or to accelerate fix-point computation in programs. Consider a loop that contains store operations. The value to be stored can be over-approximated using widening. Since mo posets contain abstracted execution histories, adding a store event in posets at least once for each store instruction within the loop will suffice to inform that the store has occurred at least once in the execution. However, one can always choose to add different events corresponding to the same store instruction depending on the precision requirement and then widen using $\nabla$, as necessary.

Note that one can use widening after analyzing some fixed $n$ iterations of a program loop. In particular, widening is applied in the transfer function for store and rmw in function SeqAI.

\section{Implementation and Evaluation}

In this section, we discuss the details of PRIORI's implementation and evaluation. In the absence of TMAI tools for RA programs, we have shown the comparison of PRIORI with the existing tools designed for the RA memory model. VBMC [1] is the most recent BMC technique among these tools. Other static tools such as CPPMEM and HERD are not designed as verification tools. CPPMEM is designed to help investigate possible ordering relations in programs under the $\mathrm{C} / \mathrm{C}++11$ memory model. It computes all the relations of all possible executions. HERD is designed to generate litmus tests for different memory models or to simulate a memory model. Both of these tools are relatively very slow compared to existing verification or bug-finding tools. We have also compared PRIORI with dynamic tools such as CDSCHECKER [28], TRACER [2], and RCMC [19] to evaluate how well PRIORI performs as a refutation tool; although the input coverage guarantee of PRIORI and dynamic checkers is quite different.

\subsection{Implementation}

PRIORI is implemented as an LLVM Compiler analysis pass written in $\mathrm{C}++$ (code size $~ 5.4 \mathrm{KLOC}$ ). PRIORI uses the APRON library [16] for manipulating the 
variable values in octagon and interval numerical abstract domains. PRIORI takes as input an LLVM IR of an RA program compiled with -01 flag, and analyzes user assertions in programs; if assertions are not provided, then it can generate the set of reachable program states at load operations for further reasoning. In addition to the transfer functions in Figure 4, PRIORI supports lock and unlock operations. PRIORI currently does not support dynamic thread creation and non-integer variables. Function calls in the program are inlined.

Handling Loops: PRIORI provides support for loops in three ways: (i) by using the assume clause, (ii) by unrolling the loops, and (iii) by a combination of assume clause and loop unrolling. The assume clause is useful in modeling spinwait loops in programs. The option of unrolling loops is used when either the assume clause is inadequate (such as in non-terminating loops), or when we have a fixed number of iterations in the loop (such as counting loops).

Experimental setup: We have used Ubuntu 16.04 machine with Intel(R) $\mathrm{Xeon}(\mathrm{R}) 3.60 \mathrm{GHz} \mathrm{CPU}$ and $32 \mathrm{~GB}$ of RAM. The listed analysis time for each benchmark is an average of four runs. The analysis times reported are in seconds.

\subsection{Summary of Benchmarks}

Benchmarks from Tracer: The benchmarks from Tracer [2] are known to have no assertion violations. We craft an unfenced version of the dijkstra benchmark to introduce assertion-violating behaviors in it. $\mathrm{CO}-2+2 \mathrm{~W}$ benchmark has no interferences; we use this benchmark to distinguish the performance of interference-based PRIORI and non-interference-based VBMC and POET. The benchmark fibonacci has a high number of load and store operations, and is used to stress-test interference-based techniques.

Benchmarks from VBMC: The benchmarks from VBMC [1] are divided into two categories: (i) the first category has benchmarks with assertion violations with respect to the RA memory model, and (ii) the second category consists the same benchmarks with appropriate fences inserted to ensure mutual exclusion under RA semantics.

Driver Benchmarks: The benchmarks ib700wdt and keybISR are Linux device drivers taken from $[22,21,9]$. We have modified these benchmarks to use C11 constructs. The program ib700wdt simulates multiple writers accessing a buffer and one closer that closes the buffer. The benchmark keybISR is an interrupt service routine for the keyboard.

\subsection{Observations}

Comparison of PRIORI with VBMC: Tables 1 and 2 show the performance comparison of PRIORI and VBMC for discovering assertion violations and proving programs correct, respectively. VBMC with the view-bound of two, which is the same bound used in [1], is insufficient to prove the properties in the program correct. We increase the view bound one at a time and report the cumulative 
Table 1: Comparison for Bug Hunting

\begin{tabular}{|c|lr|lr|l|l|c|}
\hline \multirow{2}{*}{ Name } & \multicolumn{2}{|l|}{ PRIORI } & \multicolumn{2}{|c|}{ VBMC } & \multirow{2}{*}{ CDS } & TRACER & \multirow{2}{*}{ RCMC } \\
\cline { 2 - 5 } & T & \#It & T & VS & & & \\
\hline peterson3 & 0.12 & 3 & 0.55 & 3 & 0.01 & 0.01 & 0.05 \\
10R1W & 0.02 & 2 & 3.99 & 10 & 0.01 & 0.01 & 0.03 \\
15R1W & 0.03 & 2 & 24.45 & 15 & 0.02 & 0.01 & 0.03 \\
szymanski(7) & 0.06 & 1 & 6.58 & 2 & TO & TO & TO \\
fmax $(2,7)$ & 1.00 & 2 & $\times$ & - & 0.15 & 0.05 & TO \\
\hline
\end{tabular}

TO: Timeout $(10 \mathrm{~min}), \times$ : Did not run

Table 2: Comparison for Proof of Correctness.

\begin{tabular}{|c|cc|c|c|c|c|}
\hline \multirow{2}{*}{ Name } & \multicolumn{2}{|c|}{ PRIORI } & VBMC & \multirow{2}{*}{ CDS } & \multirow{2}{*}{ TRACER } & RCMC \\
\cline { 2 - 6 } & T & \#It & T & & & \\
\hline CO-2+2W(5) & 0.01 & 3 & 0.32 & 0.01 & 0.01 & 17.26 \\
CO-2+2W(15) & 0.02 & 3 & 1.29 & 0.02 & 0.01 & TO \\
dijkstra_fen & 0.10 & 5 & $206.70^{\dagger}$ & 0.01 & 0.01 & 0.03 \\
burns_fen & 0.02 & 4 & $37.37^{\dagger}$ & 0.02 & 0.01 & 0.02 \\
peterson_fen & 0.10 & 6 & $44.12^{\dagger}$ & 0.02 & 0.01 & 0.03 \\
tbar & 0.04 & 6 & 18.58 & 0.02 & 0.01 & 0.14 \\
hehner_c11 & 0.03 & 6 & $107.16^{\dagger}$ & 0.07 & 0.02 & 0.04 \\
red_co_20 & 0.04 & 3 & 31.47 & 23.32 & 0.13 & TO \\
exp_bug_6 & 0.45 & 6 & $\times$ & 97.13 & 0.96 & 37.82 \\
exp_bug_9 & 0.57 & 6 & $\times$ & TO & 2.98 & 437.47 \\
stack_true(12) & 0.06 & 4 & $\times$ & TO & 589.81 & TO \\
ib700wdt (1) & 0.01 & 3 & 31.73 & 0.01 & 0.01 & 0.02 \\
ib700wdt (20) & 0.05 & 3 & TO & 0.01 & 0.01 & TO \\
ib700wdt (40) & 0.07 & 3 & TO & 0.01 & 0.01 & TO \\
keybISR & 0.01 & 4 & 0.01 & 0.01 & 0.01 & 0.03 \\
fibonacci & $0.11^{\dagger}$ & 5 & 310.75 & TO & 56.4 & 20.61 \\
lamport_fen & $0.17^{\dagger}$ & 4 & 431.40 & 0.09 & 0.03 & 0.04 \\
\hline lFalse positive & TO: Timeout $(10$ min $), \times:$ Did not run
\end{tabular}

time. PRIORI found the assertion violations in benchmarks of Table 1 in better time than VBMC. It is worth noting that in peterson3, 10R1W, and 15R1W, VBMC could not find the violation with the tool's default bound of two.

The results of VBMC can be considered proof only if view bounding is relaxed and the unwiding assertions (in CBMC) hold. However, we could not find an option in VBMC to disable view bounding. Thus, we made a decision to run VBMC with a view-bound of 500 (assuming it to be sufficiently large) for the benchmarks in Table 2. The results in Table 2 illustrate that the runtimes of PRIORI are consistently better than that of VBMC. VBMC was unable to analyze benchmarks marked with $\times$, since they have mutex lock/unlock operations.

Many of the mutual exclusion benchmarks have fences, which are implemented with rmw operations. These rmw operations are critical in order to prove the property. As a matter of fact, PRIORI produces false positives without the 
improvements discussed in §7.2. Identifying rmw operations as critical operations and not deleting older $s b$-ordered rmw operations enables PRIORI to attain the sought precision.

False positives in PRIORI. The last two rows in Table 2 shows the false positive results produced by PRIORI. Our technique combines the states of different executions (having the same abstract modification order) into a single abstracted program state. This results in an over-approximation of values leading to the observed false positives in fibonacci and lamport_fen benchmarks. For instance, the false positive in lamport_fen is caused by two different branch conditions (which cannot be true simultaneously in any concrete state) evaluating to true under the abstracted program states.

Comparison of PRIORI with dynamic tools: The results in Table 1 indicate that PRIORI performs competitively or faster than dynamic tools on these benchmarks. Evidently, most of the executions of these benchmarks are buggy. Hence, the probability of dynamic analyses finding the first explored execution to be buggy is very high, leading to their considerably fast analysis times. The results in Table 2 show the analysis time over non-buggy benchmarks.

Comparison of PRIORI with Poet: POET is unsound under the RA model and reports false negatives in most of the benchmarks from Table 1. The elapsed time when PoET produced sound results is as follows: (i) TO for POET on on 10R1W and 15R1W while PRIORI analyzes them in $\sim 0.03 s$, and (ii) POET takes $80.43 \mathrm{~s}$ seconds on $\mathrm{fmax}(2,7)$, while PRIORI analyzes the benchmark in $\sim 1 s$.

\section{Conclusions}

We have presented a thread modular analysis technique for RA programs that uses partial orders over the set of totally ordered stores as abstract domains. We showed that the abstract domain forms a complete lattice and further established a Galois correspondence between the set of modification orders and the abstract domain. By forgetting the $s b$-ordered older stores, we provided a sound overapproximation on the abstract domain, which is shown to be sound for RA programs. We implemented our proposal in a tool called PRIORI, and demonstrated its effectiveness in not only finding bugs, but also for proving program properties. Our experimental results revealed that PRIORI attains a high degree of precision with significantly low analysis runtimes in comparison to other tools for RA concurrency.

Acknowledgment We thank Sanjana Singh for her help during initial discussions. This work is partially supported by the Department of Science and Technology under the grant number DST ECR/2017/003427.

\section{References}

1. Abdulla, P.A., Arora, J., Atig, M.F., Krishna, S.: Verification of programs under the release-acquire semantics. In: Proceedings of the 
40th ACM SIGPLAN Conference on Programming Language Design and Implementation. pp. 1117-1132. PLDI 2019, ACM, New York, NY, USA (2019). https://doi.org/10.1145/3314221.3314649, http://doi.acm.org/10.1145/3314221.3314649

2. Abdulla, P.A., Atig, M.F., Jonsson, B., Ngo, T.P.: Optimal stateless model checking under the release-acquire semantics. Proceedings of the ACM on Programming Languages 2(OOPSLA), 135 (2018)

3. Alglave, J., Kroening, D., Tautschnig, M.: Partial orders for efficient bounded model checking of concurrent software. In: Sharygina, N., Veith, H. (eds.) Computer Aided Verification - 25th International Conference, CAV 2013, Saint Petersburg, Russia, July 13-19, 2013. Proceedings. Lecture Notes in Computer Science, vol. 8044, pp. 141-157. Springer (2013). https://doi.org/10.1007/978-3-642-39799-8_9, https://doi.org/10.1007/978-3-642-39799-8_9

4. Batty, M., Owens, S., Sarkar, S., Sewell, P., Weber, T.: Mathematizing C++ concurrency. In: Proceedings of the 38th Annual ACM SIGPLAN-SIGACT Symposium on Principles of Programming Languages. POPL '11, ACM (2011). https://doi.org/10.1145/1926385.1926394

5. Clarke, E.M., Grumberg, O., Kroening, D., Peled, D.A., Veith, H.: Model Checking. MIT Press, Cambridge, MA, USA (2018)

6. Cousot, P., Cousot, R.: Comparing the galois connection and widening/narrowing approaches to abstract interpretation. In: Bruynooghe, M., Wirsing, M. (eds.) Programming Language Implementation and Logic Programming. Springer Berlin Heidelberg, Berlin, Heidelberg (1992)

7. Cousot, P., Cousot, R.: Abstract interpretation: Past, present and future. In: Proceedings of the Joint Meeting of the Twenty-Third EACSL Annual Conference on Computer Science Logic (CSL) and the TwentyNinth Annual ACM/IEEE Symposium on Logic in Computer Science (LICS). CSL-LICS '14, Association for Computing Machinery, New York, NY, USA (2014). https://doi.org/10.1145/2603088.2603165, https://doi.org/10.1145/2603088.2603165

8. Doko, M., Vafeiadis, V.: A program logic for c11 memory fences. In: Proceedings of the 17th International Conference on Verification, Model Checking, and Abstract Interpretation - Volume 9583. pp. 413-430. VMCAI 2016, Springer-Verlag New York, Inc., New York, NY, USA (2016). https://doi.org/10.1007/978-3-662-49122-5_20, http://dx.doi.org/10.1007/978-3-662-49122-5_20

9. Farzan, A., Kincaid, Z.: Duet: Static Analysis for Unbounded Parallelism. In: Computer Aided Verification. pp. 191-196. Springer, Berlin, Heidelberg (Jul 2013). https://doi.org/10.1007/978-3-642-39799-8_12, https://link. springer. com/chapter/10.1007/978-3-642-39799-8_12

10. Flanagan, C., Qadeer, S.: Thread-modular model checking. In: Ball, T., Rajamani, S.K. (eds.) Model Checking Software. pp. 213-224. Springer Berlin Heidelberg (2003)

11. Forejt, V., Joshi, S., Kroening, D., Narayanaswamy, G., Sharma, S.: Precise predictive analysis for discovering communication deadlocks in MPI programs. ACM Trans. Program. Lang. Syst. 39(4), 15:1-15:27 (2017). https://doi.org/10.1145/3095075, https://doi.org/10.1145/3095075

12. Gavrilenko, N., Ponce-de León, H., Furbach, F., Heljanko, K., Meyer, R.: BMC for weak memory models: Relation analysis for compact SMT encodings. In: Computer Aided Verification. Springer International Publishing (2019) 
13. Henzinger, T.A., Ranjit, J., Majumdar, R., Qadeer, S.: Thread-modular abstraction refinement. In: Computer Aided Verification. Springer Berlin Heidelberg (2003)

14. Huang, S., Huang, J.: Maximal causality reduction for TSO and PSO. In: Visser, E., Smaragdakis, Y. (eds.) Proceedings of the 2016 ACM SIGPLAN International Conference on Object-Oriented Programming, Systems, Languages, and Applications, OOPSLA 2016, part of SPLASH 2016, Amsterdam, The Netherlands, October 30 - November 4, 2016. pp. 447-461. ACM (2016). https://doi.org/10.1145/2983990.2984025, https://doi.org/10.1145/2983990.2984025

15. ISO/IEC-JTC1/SC22/WG21: Programming languages - C++ (2013), http://www.open-std.org/jtc1/sc22/wg21/docs/papers/2013/n3690.pdf

16. Jeannet, B., Miné, A.: Apron: A library of numerical abstract domains for static analysis. In: Bouajjani, A., Maler, O. (eds.) Computer Aided Verification. pp. 661667. Springer Berlin Heidelberg, Berlin, Heidelberg (2009)

17. Kähkönen, K., Saarikivi, O., Heljanko, K.: Unfolding based automated testing of multithreaded programs. Automated Software Engg. 22(4), 475-515 (Dec 2015). https://doi.org/10.1007/s10515-014-0150-6, https://doi.org/10.1007/s10515-014-0150-6

18. Kang, J., Hur, C.K., Lahav, O., Vafeiadis, V., Dreyer, D.: A promising semantics for relaxed-memory concurrency. In: Proceedings of the 44th ACM SIGPLAN Symposium on Principles of Programming Languages. POPL 2017 (2017). https://doi.org/10.1145/3009837.3009850, http://doi.acm.org/10.1145/3009837.3009850

19. Kokologiannakis, M., Lahav, O., Sagonas, K., Vafeiadis, V.: Effective stateless model checking for $\mathrm{C} / \mathrm{C}++$ concurrency. Proc. ACM Program. Lang. 2(POPL), 17:1-17:32 (Dec 2017). https://doi.org/10.1145/3158105, http://doi.acm.org/10.1145/3158105

20. Kokologiannakis, M., Raad, A., Vafeiadis, V.: Model checking for weakly consistent libraries. In: McKinley, K.S., Fisher, K. (eds.) Proceedings of the 40th ACM SIGPLAN Conference on Programming Language Design and Implementation, PLDI 2019, Phoenix, AZ, USA, June 22-26, 2019. pp. 96-110. ACM (2019). https://doi.org/10.1145/3314221.3314609, https://doi.org/10.1145/3314221.3314609

21. Kusano, M., Wang, C.: Flow-sensitive composition of thread-modular abstract interpretation. In: Proceedings of the 2016 24th ACM SIGSOFT International Symposium on Foundations of Software Engineering. FSE 2016, ACM (2016). https://doi.org/10.1145/2950290.2950291, http://doi.acm.org/10.1145/2950290.2950291

22. Kusano, M., Wang, C.: Thread-modular static analysis for relaxed memory models. In: Proceedings of the 2017 11th Joint Meeting on Foundations of Software Engineering. pp. 337-348. ESEC/FSE 2017, ACM, New York, NY, USA (2017). https://doi.org/10.1145/3106237.3106243, http://doi.acm.org/10.1145/3106237.3106243

23. Lahav, O., Giannarakis, N., Vafeiadis, V.: Taming release-acquire consistency. In: Proceedings of the 43rd Annual ACM SIGPLAN-SIGACT Symposium on Principles of Programming Languages. pp. 649-662. POPL '16, ACM, New York, NY, USA (2016). https://doi.org/10.1145/2837614.2837643, http://doi.acm.org/10.1145/2837614.2837643

24. Lahav, O., Vafeiadis, V.: Owicki-gries reasoning for weak memory models. In: Automata, Languages, and Programming. Springer Berlin Heidelberg (2015) 
25. Lahav, O., Vafeiadis, V., Kang, J., Hur, C.K., Dreyer, D.: Repairing sequential consistency in $\mathrm{C} / \mathrm{C}++11$. In: Proceedings of the 38th ACM SIGPLAN Conference on Programming Language Design and Implementation. PLDI 2017 (2017). https://doi.org/10.1145/3062341.3062352, http://doi.acm.org/10.1145/3062341.3062352

26. Monat, R., Miné, A.: Precise thread-modular abstract interpretation of concurrent programs using relational interference abstractions. In: Verification, Model Checking, and Abstract Interpretation. Springer International Publishing (2017)

27. Nielson, F., Nielson, H.R., Hankin, C.: Principles of Program Analysis. Springer (2010)

28. Norris, B., Demsky, B.: A practical approach for model checking $\mathrm{C} / \mathrm{C}++11$ code. ACM Trans. Program. Lang. Syst. 38(3), 10:1-10:51 (2016)

29. Rodríguez, C., Sousa, M., Sharma, S., Kroening, D.: Unfolding-based partial order reduction. In: Aceto, L., de Frutos-Escrig, D. (eds.) 26th International Conference on Concurrency Theory, CONCUR 2015, Madrid, Spain, September 1.4, 2015. LIPIcs, vol. 42, pp. 456-469. Schloss Dagstuhl - Leibniz-Zentrum für Informatik (2015). https://doi.org/10.4230/LIPIcs.CONCUR.2015.456, https://doi.org/10.4230/LIPIcs.CONCUR.2015.456

30. Sousa, M., Rodríguez, C., D’Silva, V., Kroening, D.: Abstract interpretation with unfoldings. In: Majumdar, R., Kuncak, V. (eds.) Computer Aided Verification - 29th International Conference, CAV 2017, Heidelberg, Germany, July 2428, 2017, Proceedings, Part II. Lecture Notes in Computer Science, vol. 10427, pp. 197-216. Springer (2017). https://doi.org/10.1007/978-3-319-63390-9_11, https://doi.org/10.1007/978-3-319-63390-9_11

31. Suzanne, T., Miné, A.: From array domains to abstract interpretation under storebuffer-based memory models. In: Static Analysis. Springer Berlin Heidelberg (2016)

32. Suzanne, T., Miné, A.: Relational thread-modular abstract interpretation under relaxed memory models. In: Ryu, S. (ed.) Programming Languages and Systems. Springer International Publishing (2018)

33. Tassarotti, J., Dreyer, D., Vafeiadis, V.: Verifying read-copy-update in a logic for weak memory. In: Proceedings of the 36th ACM SIGPLAN Conference on Programming Language Design and Implementation. p. 110-120. PLDI '15, Association for Computing Machinery, New York, NY, USA (2015). https://doi.org/10.1145/2737924.2737992, https://doi.org/10.1145/2737924.2737992

34. Turon, A., Vafeiadis, V., Dreyer, D.: Gps: Navigating weak memory with ghosts, protocols, and separation. In: Proceedings of the 2014 ACM International Conference on Object Oriented Programming Systems Languages \& Applications. pp. 691-707. OOPSLA '14, ACM, New York, NY, USA (2014). https://doi.org/10.1145/2660193.2660243, http://doi.acm.org/10.1145/2660193.2660243

35. Vafeiadis, V., Narayan, C.: Relaxed separation logic: A program logic for c11 concurrency. In: Proceedings of the 2013 ACM SIGPLAN International Conference on Object Oriented Programming Systems Languages \& Applications. pp. 867-884. OOPSLA '13, ACM, New York, NY, USA (2013). https://doi.org/10.1145/2509136.2509532, http://doi.acm.org/10.1145/2509136.2509532

36. Wang, C., Kundu, S., Ganai, M.K., Gupta, A.: Symbolic predictive analysis for concurrent programs. In: Cavalcanti, A., Dams, D. (eds.) FM 2009: Formal Methods, Second World Congress, Eindhoven, The Netherlands, November 2-6, 2009. Proceedings. Lecture Notes in Computer Science, vol. 5850, 
pp. 256-272. Springer (2009). https://doi.org/10.1007/978-3-642-05089-3_17, https://doi.org/10.1007/978-3-642-05089-3_17

37. Zhang, N., Kusano, M., Wang, C.: Dynamic partial order reduction for relaxed memory models. In: Grove, D., Blackburn, S. (eds.) Proceedings of the 36th ACM SIGPLAN Conference on Programming Language Design and Implementation, Portland, OR, USA, June 15-17, 2015. pp. 250-259. ACM (2015). https://doi.org/10.1145/2737924.2737956, https://doi.org/10.1145/2737924.2737956 


\section{A Examples explaining $\mathrm{VBMC}$ and FruitTree}

\section{A.1}

$$
\begin{gathered}
x_{\text {init }}:=0 \\
a: x:=1 \stackrel{\mathrm{rf}}{\rightarrow} b: r_{1}:=x \quad d: r_{2}:=x \\
\mathrm{sb} \downarrow \\
c: x:=2 \text { - } \mathrm{rf} \quad \text { e } \quad \text { sb } \\
\mathrm{h} b r_{3}:=x \\
P: r_{1}=1 \wedge r_{2}=2 \Longrightarrow r_{3}=2 \\
\text { (Trans-Dep) }
\end{gathered}
$$

Fig. 5: HB relation via transitivity in (Trans-Dep)

Consider the example (Trans-Dep) shown in Figure 5. Property $P$ in the example can be proved only if the inter-thread dependencies are captured. There is an ordering relation from $a$ to $e$ when $r_{1}=1 \wedge r_{2}=2$, which is necessary to prove $P$. FruitTree is unable to show $P$ is valid as it does not compute transitive inter-thread dependencies beyond two threads.

\section{A.2}

Consider the example shown in (Modified-1W2R).

$$
\begin{gathered}
\text { (Modified-1W2R) } \\
a: x:=1\left\|\begin{array}{l}
b: r_{1}:=x \| \\
c: y:=r_{1} \|
\end{array}\right\| r_{2}:=x \\
P: r_{3}:=y \\
\left(x_{0}, y_{0}\right) \\
a:\left(x_{1}, y_{0}\right) \quad b \quad r_{3} \neq 1
\end{gathered}
$$

Fig. 6: Program (above); View-switches (below)

The illustration below shows the view-switches. The pair $\left(x_{i}, y_{j}\right)$ represents a view in which timestamps of variables $x$ and $y$ are $i$ and $j$, respectively. The column $m_{x}, m_{y}$ represents a message-pool indicating the latest value for $x, y$, 
respectively. Each store instruction adds a message to the message-pool. A load instruction reads a message from the message-pool (blue dashed edges). The number of dashed edges represents the view-switch count of the program. When thread 1 executes the instruction a, it updates its view for variable $x$, generating a new view $\left(x_{1}, y_{0}\right)$, which is added to the pool $m_{x}$. The assertion can be violated only if instruction $\mathrm{b}$ reads value 1 . Notice that with a view-switch bound set to two, VBMC fails to catch the violation of property $P$ in the program. Indeed with a view-switching bound set to three can discover the assertion violation, but adding more readers in this example will make any fixed bound insufficient.

\section{B On the Abstraction of Po Domain}
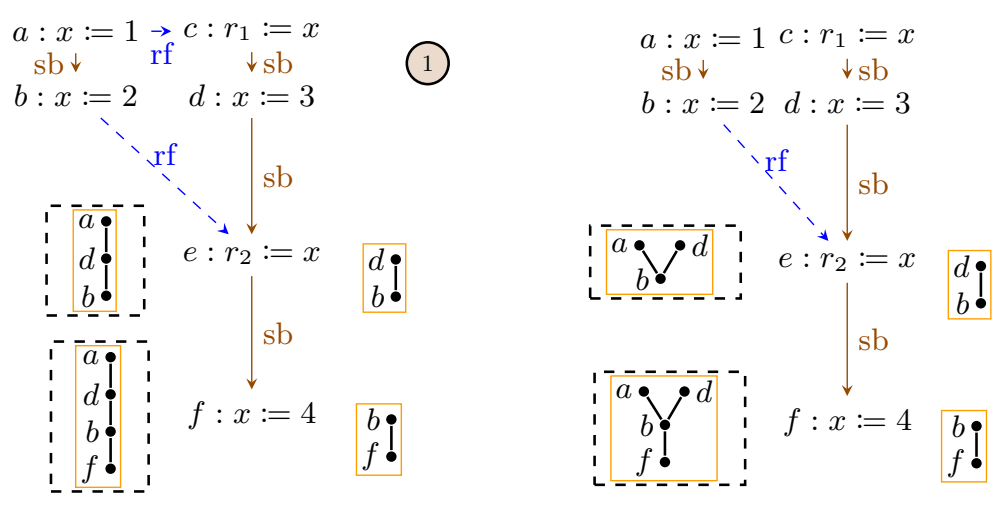

Consider execution (1). At $e$, our analysis will compute the state with $P O_{x}$ as shown on the left of $e$ when specific interferences, $a \rightarrow^{r f} c$ and $b \rightarrow^{r f} e$, are applied. Since $a \rightarrow{ }^{s b} b$, the analysis removes $a$ from the abstrct poset shown in the right of $e$. When store $f$ is analyzed, we apply similar arguments as above to obtain the poset (left) and its abstraction (right). Now consider execution (2) but with the application of single interference $b \rightarrow^{r f} e$. We observe that while $P O_{x}$ at $e$ and $f$ are different from the corressponding $P O_{x}$ in (1), the abstracted $P O_{x}$ are the same in the two executions.

\section{C mo Losets to Posets}

\section{C.1 Proofs}

Lemma 1. $(\mathcal{T}, \underline{\odot})$, is a poset.

Reflexive: Let $t \stackrel{\text { def }}{=}(S,\{\leqslant\})$ be an element in $\mathcal{T}$. Since $S \supseteq S$ and $\forall \leqslant_{i} \in\{\leqslant$ \} $\left.\exists \leqslant \leqslant_{j} \in\{\leqslant\}, \forall a, b \in S a \leqslant_{i} b \Longrightarrow a \leqslant_{j} b\right)$ is true for all $i=j$. Hence the relation $\subseteq$ is reflexive. 
Transitive: Let for some $t_{1} \stackrel{\text { def }}{=}\left(S_{1},\left\{[\leqslant\}_{1}\right), t_{2} \stackrel{\text { def }}{=}\left(S_{2},\{\leqslant \leqslant\}_{2}\right)\right.$ and $t_{3} \stackrel{\text { def }}{=}\left(S_{3},\{\leqslant \leqslant\right.$ $\left.\mathbb{J}_{3}\right)$ we have $t_{1} \subseteq t_{2}$ and $t_{2} \subseteq t_{3}$. From $t_{1} \subseteq t_{2} \Longleftrightarrow\left(S_{1} \supseteq S_{2} \wedge \forall \leqslant 1_{i} \in\{\leqslant\right.$ \}$\left._{1} \exists \leqslant 2_{2} \in\{\leqslant\}_{2} . \forall a, b \in S_{2} a \leqslant_{1_{i}} b \Longrightarrow a \leqslant_{2_{j}} b\right)$ and from $t_{2} \subseteq t_{3} \Longleftrightarrow\left(S_{2} \supseteq\right.$ $S_{3} \wedge \forall \leqslant_{2_{i}} \in\left\{\mathbb{\{} \leqslant \mathbb{\}}_{2} \exists \leqslant_{3_{j}} \in\left\{\left\{\mathbb{\}}_{3} . \forall a, b \in S_{3} a \leqslant_{2_{i}} b \Longrightarrow a \leqslant_{3_{j}} b\right)\right.\right.$. Now we have $S_{1} \supseteq S_{2}$ and $S_{2} \supseteq S_{3}$. Hence, $S_{1} \supseteq S_{3}$. Similarly, we have $\forall \leqslant 1_{1_{i}} \in\{\leqslant\}_{1} \exists \leqslant 2_{2_{j}} \in$ $\left\{\leqslant \mathbb{\}}_{2} . \forall a, b \in S_{2} a \leqslant_{1_{i}} b \Longrightarrow a \leqslant_{2_{j}} b\right.$ and $\forall \leqslant_{2_{i}} \in\left\{\leqslant \mathbb{\}}_{2} \exists \leqslant 3_{3_{j}} \in\left\{\leqslant \mathbb{\}}_{3} . \forall a, b \in\right.\right.$ $S_{3} a \leqslant 2_{2_{i}} b \Longrightarrow a \leqslant_{3_{j}} b$. Hence we have $\forall \leqslant 1_{1_{i}} \in\{\leqslant \leqslant\}_{1} \exists \leqslant 3_{j} \in\{\leqslant\}_{3} . \forall a, b \in$ $S_{3} a \leqslant_{1_{i}} b \Longrightarrow a \leqslant_{3_{j}} b$, i.e., $t_{1} \subseteq t_{3}$. Hence $\subseteq$ is a transitive relation.

Anti-symmetric: Let for some $t_{1} \stackrel{\text { def }}{=}\left(S_{1},\left\{[\leqslant\}_{1}\right)\right.$ and $t_{2} \stackrel{\text { def }}{=}\left(S_{2},\left\{[\leqslant\}_{2}\right), t_{1} \subseteq t_{2}\right.$ and $t_{2} \subseteq t_{1}$. We know that $t_{1} \subseteq t_{2} \Longleftrightarrow\left(S_{1} \supseteq S_{2} \wedge \forall \leqslant_{1_{i}} \in\{\leqslant \leqslant\}_{1} \exists \leqslant 2_{2_{j}} \in\{\leqslant\right.$ \}$\left._{2} . \forall a, b \in S_{2} a \leqslant_{1_{i}} b \Longrightarrow a \leqslant_{2_{j}} b\right)$. Now $t_{2} \subseteq t_{1} \Longleftrightarrow S_{2} \supseteq S_{1}$. Hence we have $S_{1}=S_{2}$. Now we can write $\forall \leqslant 1_{i} \in\left\{\leqslant \mathbb{\}}_{1} \exists \leqslant 2_{j} \in\{\leqslant\}_{2} . \forall a, b \in S_{2} a \leqslant 1_{i} b \Longrightarrow\right.$ $a \leqslant 2_{j} b$ as $\{\leqslant\}_{1} \subseteq\left\{\leqslant \mathbb{\}}_{2}\right.$. Similarly, from $t_{2} \subseteq t_{1}$, we get $\{\leqslant\}_{2} \subseteq\{\leqslant\}_{1}$. Which is possible only if $\left\{\leqslant \mathbb{\}}_{1}=\left\{\{\leqslant\}_{2}\right.\right.$. Hence $t_{1}=t_{2}$.

The relation $\subseteq$ is transitive, reflexive and anti-symmetric by definition. Hence $(\mathcal{T}, \subseteq)$ forms a poset.

Lemma 2. The operators $\sqcup$ and $\sqcap$ defines lub and glb of any two elements of $\mathcal{P}$, respectively.

Proof of lub: Let for any two elements $p_{1}=\left(M_{x}, \preccurlyeq 1\right)$ and $p_{2}=\left(N_{x}, \preccurlyeq 2\right)$ from set $\mathcal{P}, p=p_{1} \sqcup p_{2}$. Let $p=\left(Q_{x}, \preccurlyeq\right)$. Let $p_{a}=\left(A_{x}, \preccurlyeq a\right) \in \mathcal{P}$ be some other upper bound of the elements $p_{1}$ and $p_{2}$, i.e, $p_{1} \sqsubseteq p_{a} \wedge p_{2} \sqsubseteq p_{a}$. By definition, $p_{1} \sqsubseteq p_{a} \Longleftrightarrow$ $M_{x} \supseteq A_{x} \wedge e_{1} \preccurlyeq_{a} e_{2} \Longrightarrow e_{1} \preccurlyeq_{1} e_{2}$ and $p_{2} \sqsubseteq p_{a} \Longleftrightarrow N_{x} \supseteq A_{x} \wedge e_{1} \preccurlyeq_{a} e_{2} \Longrightarrow$ $e_{1} \preccurlyeq_{2} e_{2}$ By definition of $\sqcup$ operator, $Q_{x}=M_{x} \cap N_{x}$ and $\preccurlyeq=\preccurlyeq_{1} \cap \preccurlyeq_{2}$. Since $p_{1} \sqsubseteq p_{a} \wedge p_{2} \sqsubseteq p_{a},\left(M_{x} \supseteq A_{x} \wedge N_{x} \supseteq A_{x}\right) \wedge\left(e_{1} \preccurlyeq a e_{2} \Longrightarrow e_{1} \preccurlyeq 1 e_{2} \wedge e_{1} \preccurlyeq \preccurlyeq_{2} e_{2}\right)$. Hence, $\left(M_{x} \cap N_{x} \supseteq A_{x}\right) \wedge\left(\left(e_{1}, e_{2}\right) \in \preccurlyeq a \Longrightarrow\left(e_{1}, e_{2}\right) \in \preccurlyeq_{1} \cap \preccurlyeq_{2}\right)$. Hence $Q_{x} \supseteq A_{x} \wedge e_{1} \preccurlyeq a e_{2} \Longrightarrow e_{1} \preccurlyeq e_{2}$. Therefore, $p \sqsubseteq p_{a}$. Hence $p_{1} \sqcup p_{2}$ is the lowest upper bound of $p_{1}$ and $p_{2}$.

Proof of glb: Let for any two elements $p_{1}=\left(M_{x}, \preccurlyeq 1\right)$ and $p_{2}=\left(N_{x}, \preccurlyeq 2\right)$ from set $\mathcal{P}, p=p_{1} \sqcap p_{2}$. Let $p=\left(Q_{x}, \preccurlyeq\right)$. Let $p_{a}=\left(A_{x}, \preccurlyeq a\right) \in \mathcal{P}$ be some other lower bound of the elements $p_{1}$ and $p_{2}$, i.e, $p_{a} \sqsubseteq p_{1} \wedge p_{a} \sqsubseteq p_{2}$. By definition, $p_{a} \sqsubseteq p_{1} \Longleftrightarrow A_{x} \supseteq M_{x} \wedge e_{1} \preccurlyeq e_{1} e_{2} \Longrightarrow e_{1} \preccurlyeq a e_{2}$ and $p_{a} \sqsubseteq p_{2} \Longleftrightarrow A_{x} \supseteq$ $N_{x} \wedge e_{1} \preccurlyeq{ }_{2} e_{2} \Longrightarrow e_{1} \preccurlyeq a_{a} e_{2}$.

If $p_{1} \uparrow p_{2}$, by the definition of meet operation $Q_{x}=M_{x} \cup N_{x}$ and $\preccurlyeq=\preccurlyeq_{1} \cup \preccurlyeq_{2}$. Since $A_{x} \supseteq M_{x}$ and $A_{x} \supseteq N_{x}$, then $A_{x} \supseteq M_{x} \cup N_{x}$. Hence $A_{x} \supseteq Q_{x}$. From $e_{1} \preccurlyeq{ }_{1} e_{2} \Longrightarrow e_{1} \preccurlyeq{ }_{a} e_{2}$ and $e_{1} \preccurlyeq{ }_{2} e_{2} \Longrightarrow e_{1} \preccurlyeq{ }_{a} e_{2}$, we have $e_{1} \preccurlyeq{ }_{1} e_{2} \vee e_{1} \preccurlyeq{ }_{2} e_{2} \Longrightarrow$ $e_{1} \preccurlyeq a e_{2}$. From definition of meet operator, $e_{1} \preccurlyeq e_{2} \Longrightarrow e_{1} \preccurlyeq e_{1} e_{2} \vee e_{1} \preccurlyeq 2 e_{2}$. Hence $e_{1} \preccurlyeq e_{2} \Longrightarrow e_{1} \preccurlyeq a e_{2}$. Therefore, $A_{x} \supseteq Q_{x} \wedge e_{1} \preccurlyeq e_{2} \Longrightarrow e_{1} \preccurlyeq a_{a} e_{2}$ Hence $p_{a} \sqsubseteq p$ i.e., $p$ is greatest lower bound of $p_{1}$ and $p_{2}$.

If $p_{1} \uparrow p_{2}$ is false, the only consistent partial order $p_{a}$ such that $p_{a} \sqsubseteq p_{1} \wedge p_{a} \sqsubseteq p_{2}$, is $\perp$. Hence $p_{1} \sqcap p_{2}$ gives the greatest lower bound.

Lemma 3. ( $\mathcal{P}, \sqsubseteq, \sqcup, \sqcap, \perp, \top)$ is a complete lattice, where $\mathcal{P}$ is set of all possible partial orders over elements of set $\mathrm{St}$ and $\mathrm{T}$ is defined as empty poset and $\perp$ is a special elements in $\mathcal{P}$ that is ordered below all the elements of $\mathcal{P}$ in $\sqsubseteq$. 
The relation $\sqsubseteq$ is transitive, reflexive and anti-symmetric by definition. Hence $(\mathcal{P}, \sqsubseteq)$ is a poset. Lemma 2 proves that the operators $\sqcup$ and $\sqcap$ compute a $l u b$ and $g l b$, respectively, of any two elements of $\mathcal{P}$. Hence, $(\mathcal{P}, \sqsubseteq, \sqcup, \sqcap, \perp, \top)$ is a lattice. To prove that it is a complete lattice, we need to prove that for every $P \subset \mathcal{P}, \sqcup$ and $\sqcap$ over set $P$ exits.

$\sqcup$ operation over all subsets: Using the definition of $\sqcup$ operation, for some $P \subset \mathcal{P}, \sqcup P$ can be defined as $p=\left(Q_{x}, \preccurlyeq\right)$, where $Q_{x}=\bigcap_{p_{i} \in P} M(i)_{x}$ and $\preccurlyeq=$ $\bigcap_{p_{i} \in P} \preccurlyeq_{i}$, where $p_{i}=\left(M(i)_{x}, \preccurlyeq_{i}\right)$. Let $p_{a}=\left(A_{x}, \preccurlyeq_{a}\right) \in \mathcal{P}$ be an upper bound of $P$. We know $p_{a}$ exists because by definition, $\top$ is an upper bound of all the lattice elements. Since $p_{a}$ is an upper bound of $P, \forall p_{i} \in P p_{i} \sqsubseteq p_{a} \Longleftrightarrow M(i)_{x} \supseteq A_{x} \wedge$ $\left(e_{1} \preccurlyeq_{a} e_{2} \Longrightarrow e_{1} \preccurlyeq_{i} e_{2}\right)$ by definition of $\sqsubseteq$ operation. Using simple set operations, we can say that $\left(\forall p_{i} \in P M(i)_{x} \supseteq A_{x}\right) \Longrightarrow \bigcap_{p_{i} \in P} M(i)_{x} \supseteq A_{x} \Longrightarrow Q_{x} \supseteq A_{x}$. Similarly, $\left(\forall p_{i} \in P\left(e_{1}, e_{2}\right) \in \preccurlyeq_{a} \Longrightarrow\left(e_{1}, e_{2}\right) \in \preccurlyeq_{i}\right) \Longleftrightarrow\left(\left(e_{1}, e_{2}\right) \in \preccurlyeq_{a} \Longrightarrow\right.$ $\left.\left(\forall p_{i} \in P\left(e_{1}, e_{2}\right) \in \preccurlyeq_{i}\right)\right) \Longleftrightarrow\left(\left(e_{1}, e_{2}\right) \in \preccurlyeq_{a} \Longrightarrow\left(e_{1}, e_{2}\right) \in \bigcap_{p_{i} \in P} \preccurlyeq_{i}\right) \Longleftrightarrow$ $\left(\left(e_{1}, e_{2}\right) \in \preccurlyeq a \Longrightarrow\left(e_{1}, e_{2}\right) \in \preccurlyeq\right)$. Hence $p \sqsubseteq p_{a}$.

$\sqcap$ operation over all subsets: Let for some $P \subset \mathcal{P}, \sqcap$ over set $P$ can be defined as $p=\left(Q_{x}, \preccurlyeq\right)$ if $\uparrow P$, then $Q_{x}=\bigcup_{p_{i} \in P} M(i)_{x}$ and $\preccurlyeq=\bigcup_{p_{i} \in P} \preccurlyeq_{i}$ else $\perp$. If $\uparrow P$ is false, the only possible lower bound of $P$ is $\perp$. Hence, we need to prove the existence of glb only if $\uparrow P$. Let $p_{a}=\left(A_{x}, \preccurlyeq a\right) \in \mathcal{P}$ be a lower bound of $P$. We know $p_{a}$ exists because by definition, $\perp$ is a lower bound of all the lattice elements. Since $p_{a}$ is a lower bound of $P, \forall p_{i} \in P \forall p_{i} \in P, p_{a} \sqsubseteq p_{i}$. Therefore, $\left(\forall p_{i} \in P, A_{x} \supseteq M(i)_{x}\right) \Longleftrightarrow A_{x} \supseteq \bigcup_{p_{i} \in P} M(i)_{x} \Longleftrightarrow A_{x} \supseteq Q_{x}$. Similarly, from $\forall p_{i} \in P, p_{a} \sqsubseteq p_{i}$, we have $\left(\forall p_{i} \in P,\left(e_{1}, e_{2}\right) \in \preccurlyeq{ }_{i} \Longrightarrow\left(e_{1}, e_{2}\right) \in \preccurlyeq_{a}\right) \Longleftrightarrow$ $\left(\left(e_{1}, e_{2}\right) \in \bigcup_{p_{i} \in P} \preccurlyeq i \Longrightarrow\left(e_{1}, e_{2}\right) \in \preccurlyeq a\right) \Longleftrightarrow\left(\left(e_{1}, e_{2}\right) \in \preccurlyeq \Longrightarrow\left(e_{1}, e_{2}\right) \in \preccurlyeq a\right)$. Now we have $A_{x} \supseteq Q_{x} \wedge\left(\left(e_{1}, e_{2}\right) \in \preccurlyeq \Longrightarrow\left(e_{1}, e_{2}\right) \in \preccurlyeq a\right)$. Hence, $p_{a} \sqsubseteq p$, which proves that $p$ is glb of set $P$.

Therefore $(\mathcal{P}, \sqsubseteq, \sqcup, \sqcap, \perp, \top)$ is a complete lattice.

Lemma 4. The operation $\nabla$ defines widening operator over elements of lattice

The operation $\nabla$ is widening operator over lattice $(\mathcal{P}, \sqsubseteq, \sqcup, \sqcap, \perp, \top)$ iff

(i) $\nabla$ is an upper-bound operator i.e, $p_{1}, p_{2} \sqsubseteq p_{1} \nabla p_{2}$, and

(ii) for all ascending chains $p_{0}, p_{1}, p_{2}, \ldots$ over elements of lattice $\mathcal{P}$, the ascending chain $p_{0}^{\nabla}, p_{1}^{\nabla}, p_{2}^{\nabla} \ldots$ eventually stabilizes, where $p_{i}^{\nabla}$ is defined as $p_{0}^{\nabla}=p_{0}$ and $\forall i>0, p_{i}^{\nabla}=p_{i-1}^{\nabla} \nabla p_{i}$.

Proof of (i) Let $p=p_{1} \nabla p_{2}$ for some $p_{1}=\left(M_{x}, \preccurlyeq 1\right), p_{2}=\left(N_{x}, \preccurlyeq_{2}\right)$ and $p=\left(Q_{x}, \preccurlyeq\right)$. By definition of $\nabla, M_{x} \supseteq Q_{x}$ and $N_{x} \supseteq Q_{x}$. Similarly, $a \preccurlyeq b \Longrightarrow$ $a \preccurlyeq_{1} b \wedge a \preccurlyeq_{2} b$. Therefore, $p_{1}, p_{2} \sqsubseteq p$. Hence $\nabla$ is an upper bound operator.

Proof of (ii) Since the number of program instructions are finite in any program, we have a finite set of instruction labels. By definition of $\nabla$, for any $p=\left(Q_{x}, \preccurlyeq\right)$ 
such that $p=p_{1} \nabla p_{2}$, the set $Q_{x}$ may contain at most one event for each instruction label. Hence in any such $p$, the set of events $Q_{x}$ is finite. There are only finitely many possible pair may exist over a finite set $Q_{x}$. Hence, the set $\preccurlyeq$ is also finite.

Therefore, we can say that in chain $p_{0}^{\nabla}, p_{1}^{\nabla}, p_{2}^{\nabla} \ldots$, where $p_{i}^{\nabla}=\left(M\left(i^{\nabla}\right)_{x}, \preccurlyeq i_{i}\right.$ ), $M\left(i^{\nabla}\right)_{x}$ and $\preccurlyeq_{i \nabla}$ are finite for all $i>0$. We know that, the chain $p_{0}^{\nabla}, p_{1}^{\nabla}, p_{2}^{\nabla} \ldots$ is an ascending chain (property of upper-bound operator [27]). Hence, from definition of $\sqsubseteq$ we have $\forall i>0, M\left(i^{\nabla}\right)_{x} \subseteq M\left(i-1^{\nabla}\right)_{x} \wedge \preccurlyeq_{i \nabla} \subseteq \preccurlyeq_{i-1} \nabla$. Let chain $p_{0}^{\nabla}, p_{1}^{\nabla}, p_{2}^{\nabla} \ldots$ does not stabilize. Hence $\forall i>0$, either $M\left(i^{\nabla}\right)_{x} \subset M\left(i-1^{\nabla}\right)_{x}$ or $\preccurlyeq_{i} \nabla \subset \preccurlyeq_{i-1} \nabla$. We can also say that $\forall i>0$ either $\left|M\left(i^{\nabla}\right)_{x}\right|<\left|M\left(i-1^{\nabla}\right)_{x}\right|$ or $\left|\preccurlyeq_{\mathrm{i} \nabla}\right|<\left|\preccurlyeq_{\mathrm{i}-1 \nabla}\right|$ where $\left|M\left(i^{\nabla}\right)_{x}\right|,\left|M\left(i-1^{\nabla}\right)_{x}\right|,\left|\preccurlyeq_{\mathrm{i} \nabla}\right|$ and $\left|\preccurlyeq_{\mathrm{i}-1} \nabla\right|$ are some natural number $<\omega$. Now, $\left|M\left(i^{\nabla}\right)_{x}\right|<\left|M\left(i-1^{\nabla}\right)_{x}\right|$ is not possible infinitely often since any strictly decreasing chain over $\left|M\left(i^{\nabla}\right)_{x}\right|$ starting from some natural number $<\omega$ will eventually reach minimal element 0 and can not decrease further. Similarly, $\left|\preccurlyeq_{\mathrm{i} \nabla}\right|<\left|\preccurlyeq_{\mathrm{i}-1 \nabla}\right|$ is not possible infinitely often. Hence a strictly decreasing chain of $\left(\left|M\left(i^{\nabla}\right)_{x}\right|,\left|\preccurlyeq_{i} \nabla\right|\right)$ form cannot be infinite. It means that a infinite strictly ascending chain over $p_{i}^{\nabla}$ is not possible. Therefore, the chain $p_{0}^{\nabla}, p_{1}^{\nabla}, p_{2}^{\nabla} \ldots$ eventually stabilizes.

Lemma 5. $\left(p_{1}, p_{2}\right) \in \beta \Longrightarrow p_{1} \sqsubseteq p_{2}$.

The property is trivially true for $p_{1}=\perp$ since $\perp$ is the least element in lattice. For $p_{1} \neq \perp$, from the definition of $\beta, M_{x} \supseteq N_{x} \wedge \preccurlyeq 2 \subseteq \preccurlyeq 1$. But $\preccurlyeq_{2} \subseteq \preccurlyeq 1$ $\Longleftrightarrow a \preccurlyeq{ }_{2} b \Longrightarrow a \preccurlyeq{ }_{1} b$, Hence, by definition of $\sqsubseteq, p_{1} \sqsubseteq p_{2}$.

Lemma 6. Abstract soundness assumption holds under $\beta$, i.e., $\forall p, p_{1}, p_{2} \in \mathcal{P}$. $\left(p, p_{1}\right) \in \beta \wedge p_{1} \sqsubseteq p_{2} \Longrightarrow\left(p, p_{2}\right) \in \beta$

By Lemma 5 , we know that $\left(p, p_{1}\right) \in \beta \Longrightarrow p \sqsubseteq p_{1}$. If $p_{1}=\perp, p \sqsubseteq p_{1}$ is possible if and only if $p=\perp$. By definition of $\beta, \forall p_{2} \in \mathcal{P},\left(\perp, p_{2}\right) \in \beta$. Now for $p \neq \perp$, by definition, $\left(p, p_{1}\right) \in \beta \Longleftrightarrow\left(M_{x} \subseteq Q_{x} \backslash\left\{a \mid \exists b \in Q_{x} . a \rightarrow{ }^{s b} b \wedge a \neq b\right\} \wedge \preccurlyeq 1 \subseteq \preccurlyeq\right)$. From $p_{1} \sqsubseteq p_{2}$, it follows that $N_{x} \subseteq M_{x} \wedge \preccurlyeq 2 \subseteq \preccurlyeq 1$. Hence, we have $N_{x} \subseteq M_{x} \subseteq$ $Q_{x} \backslash\left\{a \mid \exists b \in Q_{x} \cdot a \rightarrow{ }^{s b} b \wedge a \neq b\right\}$ and $\preccurlyeq{ }_{2} \subseteq \preccurlyeq{ }_{1} \subseteq \preccurlyeq$. Hence $\left(p, p_{2}\right) \in \beta$

Lemma 7. For some set of orderings $O_{1}$ and $O_{2}$ over elements from set $S$, $O_{1} \subseteq O_{2} \Longleftrightarrow L\left(S, O_{1}\right) \supseteq L\left(S, O_{2}\right)$.

$(\Longrightarrow)$ Let for some set $S$ and ordering relations $O_{1} \subseteq O_{2}, L\left(S, O_{1}\right) \nsupseteq L\left(S, O_{2}\right)$. Without loss of generality, let $\leqslant$ be an ordering relation such that $\leqslant \notin L\left(S, O_{1}\right) \wedge$ $\leqslant \in L\left(S, O_{2}\right)$. By definition, $\leqslant$ must satisfy all the ordering defined in $\mathrm{O}_{2}$. Since $O_{1} \subseteq O_{2}$, $\leqslant$ also satisfies all the ordering in $O_{1}$. Hence $\leqslant \in L\left(S, O_{1}\right)$. Therefore, $L\left(S, O_{1}\right) \supseteq L\left(S, O_{2}\right)$.

$\left(\Longleftarrow\right.$ ) Let us assume that for some $L\left(S, O_{1}\right) \supseteq L\left(S, O_{2}\right), O_{1} \nsubseteq O_{2}$. It means all $\leqslant \in L\left(S, O_{2}\right)$ also satisfies ordering relations in $O_{1}$. Hence $O_{1} \subseteq O_{2}$.

Theorem 1. $(\mathcal{T}, \subseteq) \underset{\alpha}{\leftrightarrows}(\mathcal{P}, \sqsubseteq, \sqcup, \sqcap, \perp, \top)$ 
Let $t_{1}, t_{2} \in \mathcal{T}$, and $p_{1}, p_{2} \in \mathcal{P}$, for some $t_{1}=\left(S_{1},\{\leqslant\}_{1}\right), t_{2}=\left(S_{2},\left\{\leqslant \mathbb{\}}_{2}\right), p_{1}=\right.$ $\left(M_{x}, \preccurlyeq_{1}\right)$ and $p_{2}=\left(N_{x}, \preccurlyeq_{2}\right)$. To prove that $\alpha$ is monotonic, let $\alpha\left(t_{1}\right)=p_{1}, \alpha\left(t_{2}\right)=$ $p_{2}$, and $t_{2} \subseteq t_{1}$ without loss of generality.

$$
\begin{array}{ll}
p_{1}=\alpha\left(t_{1}\right) \Longleftrightarrow M_{x}=S_{1} \wedge \preccurlyeq \aleph_{1}=\bigcap_{i=1}^{n} \leqslant_{1_{i}} & \text { (By definition of } \alpha) \\
p_{2}=\alpha\left(t_{2}\right) \Longleftrightarrow N_{x}=S_{2} \wedge \preccurlyeq 2=\bigcap_{i=1}^{m} \leqslant_{2_{i}} & \text { (By definition of } \alpha)
\end{array}
$$

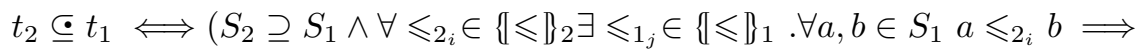

$$
\begin{aligned}
& \left.a \leqslant 1_{j} b\right) \\
& \text { (By definition of } \subseteq \text { ) } \\
& \Longleftrightarrow\left(N_{x} \supseteq M_{x} \wedge \forall \leqslant 2_{2_{i}} \in\left\{\mathbb{\{}_{\}_{2}} \exists \leqslant 1_{1_{j}} \in\left\{\mid \leqslant \mathbb{\}}_{1} . \forall a, b \in S_{1} a \leqslant 2_{2_{i}} b \Longrightarrow\right.\right.\right. \\
& \left.a \leqslant 1_{j} b\right) \\
& \text { (By eq } 1 \& 2 \text { ) } \\
& \Longleftrightarrow\left(N_{x} \supseteq M_{x} \wedge \bigcap_{i=1}^{n} \leqslant 1_{i} \subseteq \bigcap_{i=1}^{m} \leqslant 2_{i}\right) \\
& \Longleftrightarrow\left(N_{x} \subseteq M_{x} \wedge \forall a, b \in M_{x} \cdot a \preccurlyeq 1 b \Longrightarrow a \preccurlyeq \preccurlyeq_{2} b\right) \\
& \Longleftrightarrow\left(p_{2} \sqsubseteq p_{1}\right)
\end{aligned}
$$

Hence $\alpha$ is monotonic. To prove that $\gamma$ is monotonic, let $\gamma\left(p_{1}\right)=t_{1}, \gamma\left(p_{2}\right)=t_{2}$, and $p_{2} \sqsubseteq p_{1}$ without loss of generality.

$$
\begin{aligned}
& t_{1}=\gamma\left(p_{1}\right) \Longleftrightarrow S=Q_{x} \wedge\left\{\leqslant \mathbb{\}}_{1}=L\left(S_{1}, \preccurlyeq 1\right) \quad \text { (By definition of } \gamma\right. \text { ) } \\
& t_{2}=\gamma\left(p_{2}\right) \Longleftrightarrow S=Q_{x} \wedge\{\leqslant\}_{2}=L\left(S_{2}, \preccurlyeq 2\right) \quad \text { (By definition of } \gamma \text { ) }
\end{aligned}
$$

$$
\begin{array}{rlr}
p_{2} \sqsubseteq p_{1} & \Longleftrightarrow\left(N_{x} \supseteq M_{x} \wedge \preccurlyeq 1 \subseteq \preccurlyeq 2\right) & \text { (By definition of } \sqsubseteq) \\
& \Longleftrightarrow\left(S_{2} \supseteq S_{1} \wedge \preccurlyeq \preccurlyeq_{1} \subseteq \preccurlyeq_{2}\right) & \text { (By eq } 3 \& 4) \\
& \left(S_{2} \supseteq S_{1} \wedge L\left(S_{1}, \preccurlyeq 1\right) \supseteq L\left(S_{1}, \preccurlyeq 2\right)\right) & (\text { By lemma } 7) \\
& \left(S_{2} \supseteq S_{1} \wedge \forall \leqslant_{2_{i}} \in\left\{\leqslant \mathbb{\}}_{2} \exists \leqslant_{1_{j}} \in\{\leqslant\}_{1} . \forall a, b \in S_{1} a \leqslant_{2_{i}} b \Longrightarrow\right.\right. \\
& \left.a \leqslant_{1} b\right) & \\
& \Longleftrightarrow t_{2} \subseteq t_{1} & \text { (By definition of } \subseteq \text { ) }
\end{array}
$$


Hence $\gamma$ is monotonic. Let $\alpha\left(t_{1}\right)=p_{1}, \gamma\left(p_{2}\right)=t_{2}$. If we can prove that $p_{2} \sqsubseteq$ $p_{1} \Longleftrightarrow t_{2} \subseteq t_{1},(\alpha, \gamma)$ forms a Galois connection between the lattices.

$$
\begin{aligned}
& \left.p_{2} \sqsubseteq p_{1} \Longleftrightarrow\left(N_{x} \supseteq M_{x} \wedge a \preccurlyeq 1 b \Longrightarrow a \preccurlyeq_{2} b\right) \quad \text { (By definition of } \sqsubseteq\right) \\
& \Longleftrightarrow\left(N_{x} \supseteq M_{x} \wedge \preccurlyeq 1 \subseteq \preccurlyeq 2\right) \\
& \Longleftrightarrow\left(N_{x} \supseteq S_{1} \wedge \bigcap_{i=1}^{n} \leqslant{ }_{1_{i}} \subseteq \preccurlyeq 2\right) \\
& \left.\Longleftrightarrow\left(S_{2} \supseteq S_{1} \wedge \bigcap_{i=1}^{n} \leqslant_{1_{i}} \subseteq \preccurlyeq 2\right) \quad \text { (By eq } 4\right) \\
& \left.\Longleftrightarrow\left(S_{2} \supseteq S_{1} \wedge L\left(S_{1}, \bigcap_{i=1}^{n} \leqslant_{1}\right) \supseteq L\left(S_{2}, \preccurlyeq 2\right)\right) \quad \text { (By lemma } 7\right) \\
& \Longleftrightarrow\left(S_{2} \supseteq S_{1} \wedge \forall \leqslant 2_{2_{i}} \in\left\{\leqslant \mathbb{\}}_{2} \exists \leqslant 1_{j} \in\{\leqslant \leqslant\}_{1} . \forall a, b \in S_{1} a \leqslant 2_{i} b \Longrightarrow\right.\right. \\
& a \leqslant 1_{j} b \quad \text { (By well-formedness of } t_{1} \text { and eq } 4 \text { ) } \\
& \Longleftrightarrow t_{2} \subseteq t_{1}
\end{aligned}
$$

Theorem 2. There is a Galois connection among elements of $\prod_{x \in \mathcal{V}} \mathcal{P}_{x}$ and $\prod_{x \in \mathcal{V}} \mathcal{T}_{x}$.

A Galois connection between two posets can be lifted to a Galois connection in the Cartesian product of the posets [27], i.e., if $L_{1} \underset{\alpha_{1}}{\stackrel{\gamma_{1}}{\leftrightarrows}} M_{1}$ and $L_{2} \underset{\alpha_{2}}{\stackrel{\gamma_{2}}{\leftrightarrows}} M_{2}$, then $L_{1} \times L_{2} \underset{\alpha}{\stackrel{\gamma}{\leftrightarrows}} M_{1} \times M_{2}$, where $\alpha\left(l_{1}, l_{2}\right) \stackrel{\text { def }}{=}\left(\alpha_{1}\left(l_{1}\right), \alpha_{2}\left(l_{2}\right)\right), \gamma\left(m_{1}, m_{2}\right) \stackrel{\text { def }}{=}$ $\left(\gamma_{1}\left(m_{1}\right), \gamma_{2}\left(m_{2}\right)\right)$. We use this result to establish that the tuples of elements from $\prod_{x \in \mathcal{V}} \mathcal{P}_{x}$ in the program forms a lattice. Further, such a lattice will have Galois connection with the poset of $\prod_{x \in \mathcal{V}} \mathcal{T}_{x}$.

Lemma 8. Every concrete property has an abstraction under soundness relation $\beta$, i.e, $\forall p \in \mathcal{P}, \exists p^{\prime} \in \mathcal{P}$. $\left(p, p^{\prime}\right) \in \beta$.

If $p=\perp$, then $\left(p, p^{\prime}\right) \in \beta$ for all $p^{\prime} \in \mathcal{P}$. Hence the property is trivially true for $\perp$. For $p \neq \perp$, we know that $\emptyset \subseteq Q_{x} \backslash\left\{a \mid \exists b \in Q_{x} . a \rightarrow \rightarrow^{s b} b \wedge a \neq b\right\}$. Similarly, $\emptyset \subseteq \preccurlyeq$. Hence, there exist an element $(\emptyset, \emptyset) \in \mathcal{P}$ (i.e., $\top$ ) such that $(p, \top) \in \beta$.

The significance of Lemma 8 rests in the fact that every element in $\mathcal{P}$ has at least one over-approximation under the soundness relation $\beta$.

Theorem 3. Abstraction relation $\alpha^{\sharp}$ is minimal sound abstraction under soundness relation $\beta$, i.e., $\left(p_{1}, p_{2}\right) \in \beta \Longleftrightarrow \alpha^{\sharp}\left(p_{1}\right) \sqsubseteq p_{2}$.

( $\Longrightarrow$ ) For $p_{1}=\perp, \alpha^{\sharp}\left(p_{1}\right)=\perp$, which is the least element in the lattice. Let us now focus on $p_{1} \neq \perp$. Let $\alpha^{\sharp}\left(p_{1}\right)=p$. By definition, $Q_{x}=M_{x} \backslash\{a \mid \exists b \in$ $\left.Q_{x} . a \rightarrow^{s b} b \wedge a \neq b\right\}$. From $\left(p_{1}, p_{2}\right) \in \beta$, we have $N_{x} \subseteq M_{x} \backslash\left\{a \mid \exists b \in M_{x} . a \rightarrow{ }^{s b} b \wedge\right.$ $a \neq b\}$. Hence $N_{x} \subseteq Q_{x}$. Let $R=\left\{(a, b) \mid(a, b) \in \preccurlyeq 1 \wedge\left(a \notin Q_{x} \vee b \notin Q_{x}\right)\right\}$. By 
definition of $\alpha^{\sharp}$, we have $\alpha^{\sharp}\left(p_{1}\right)=p \Longleftrightarrow \preccurlyeq=\preccurlyeq 1 \backslash R$. By definition of $\beta$, we have, $\left(p_{1}, p_{2}\right) \in \beta \Longleftrightarrow \preccurlyeq_{2} \subseteq \preccurlyeq_{1}$. To prove that $p \sqsubseteq p_{2}$, we need to prove that $\preccurlyeq_{2} \subseteq \preccurlyeq$. Let us assume that $\left(a^{\prime}, b^{\prime}\right) \notin \preccurlyeq,\left(a^{\prime}, b^{\prime}\right) \in \preccurlyeq 1$. Since $\preccurlyeq=\preccurlyeq 1 \backslash R,\left(a^{\prime}, b^{\prime}\right) \notin \preccurlyeq \Longrightarrow$ $\left(a^{\prime}, b^{\prime}\right) \in R$, which is possible only if $a^{\prime} \notin Q_{x} \vee b^{\prime} \notin Q_{x}$. This is true only if either $a^{\prime} \in\left\{a \mid \exists b \in Q_{x} . a \rightarrow^{s b} b \wedge a \neq b\right\}$ or $b^{\prime} \in\left\{a \mid \exists b \in Q_{x} . a \rightarrow^{s b} b \wedge a \neq b\right\}$. Hence, $a^{\prime} \notin N_{x}$ or $b^{\prime} \notin N_{x}$, which means $\left(a^{\prime}, b^{\prime}\right) \notin \preccurlyeq 2$. Therefore, $p \sqsubseteq p_{2}$.

( $\Longleftarrow$ ) By defintion of $\beta$, for $p_{1}=\perp, \forall p_{2} \in \mathcal{P}\left(p 1, p_{2}\right) \in \beta$. Hence it is trivially true. For $p_{1} \neq \perp, \alpha^{\sharp}\left(p_{1}\right)=p \Longleftrightarrow Q_{x}=M_{x} \backslash\left\{a \mid \exists b \in M_{x} . a \rightarrow \rightarrow^{s b} b \wedge a \neq b\right\}$. Hence $Q_{x} \subseteq M_{x} \backslash\left\{a \mid \exists b \in M_{x} . a \rightarrow^{s b} b \wedge a \neq b\right\}$. Similarly, $\preccurlyeq=\preccurlyeq 1 \backslash\{(a, b) \mid$ $\left.(a, b) \in \preccurlyeq \wedge\left(a \notin Q_{x} \vee b \notin Q_{x}\right)\right\}$. Hence $\preccurlyeq \subseteq \preccurlyeq 1$. Therefore, $\left(p_{1}, p\right) \in \beta$. By Lemma 6 and $p \sqsubseteq p_{2}$, we have $\left(p_{1}, p_{2}\right) \in \beta$.

\section{Transfer functions for lock/unlock instructions under RA}

$$
\begin{aligned}
& (\operatorname{pre}(\ell), m o, m) \in \mathcal{S} \quad \ell_{l}=\operatorname{FindLock}(\ell) \\
& \frac{\ell_{l} \in \operatorname{Lasts}(m o) \quad m o^{\prime}=m o[x \rightarrow m o(l) \diamond \ell]}{\mathcal{S} \stackrel{\ell: \text { unlock } l}{\longrightarrow} \mathcal{S} \uplus\left(\ell, m o^{\prime}, m^{\prime}\right)} \text { UNLOCK } \\
& \sigma=\operatorname{PreProcessLock}(\ell) \quad \ell_{u l} \in I(\ell) \\
& \left(\ell_{u l}, m o_{u l}, m_{u l}\right) \in \mathcal{S} \quad(\text { pre }(\ell), m o, m) \in \sigma \\
& \left.\left(\operatorname{pre}(\ell), m o^{\prime \prime}, m^{\prime}\right)\right)=\mathrm{AI}\left((\operatorname{pre}(\ell), m o, m),\left(\ell_{u l}, m_{u l}, m_{u l}\right)\right) \\
& \frac{m o^{\prime}=m o^{\prime \prime} \diamond \ell}{\mathcal{S} \stackrel{\ell: \text { Iock } l}{\longrightarrow} \mathcal{S} \uplus\left(\ell, m o^{\prime}, m^{\prime}\right)} \text { LOCK }
\end{aligned}
$$

Fig. 7: Transfer functions for Lock/Unlock instructions in RA programs

The rules LOCK and UNLOCK in figure 7 shows transfer functions for lock and unlock instructions over mutex variable $l$ respectively. Whenever an unlock instructions is encountered, we check that the corresponding lock instruction $\ell_{l}$ should be at the end of partial order of mutex variable $l$ in program states of location $\operatorname{pre}(\ell)$. Intuitively, it means that the lock must be acquired by current thread in all program states at program location pre $(\ell)$. We change the program state by appending the unlock instruction $\ell$ in the partial order of mutex variable $l$.

The rule for lock instruction is a bit tricky. It uses following helper functions:

Lasts $(p)$ : Returns the set of last elements in a partial order. Formally $\{a \mid \nexists b \in$ $\left.Q_{x} \cdot(a, b) \in \preccurlyeq\right\}$

EndsInLock $(m o)$ : True if the last of a partial order contains some lock instruction, otherwise false, i.e. it returns $\exists \ell_{l} \in \operatorname{Lasts}(\operatorname{mo}(l)) \wedge \ell_{l} \in \operatorname{Locks}(l)$

FindUnlock $(\ell)$ Return the unlock instruction corresponding to lock instruction $\ell$. 
FindLock $(\ell)$ Return the Lock instruction corresponding to unlock instruction $\ell$.

PreProcessLock $(\ell)$ The function first checks if in some program state at pre $(\ell)$ the mutex variable $l$ is already acquired by some thread. This can be done by checking if partial order of mutex variable $l$ ends with a lock instruction $\ell_{l}$. If it ends in some other lock instruction $\ell_{l}$, PreProcessLock finds the unlock instructions $\ell_{u l}$ corresponding to $\ell_{l}$ and apply interference from this. It combines the resulting program states with the program state at pre $(\ell)$ that do not end in lock instruction. i.e. PreProcessLock $(\ell)$ returns $\sigma$, where $\sigma \stackrel{\text { def }}{=} \forall(\operatorname{pre}(\ell), m o, m) \in \mathcal{S}$ if $\operatorname{Ends} \operatorname{InLock}(m o)$, then $\ell_{u l}=\operatorname{FindUnlock}\left(\ell_{l}\right)$, $\forall\left(\ell_{u l}, m o_{u l}, m_{u l}\right) \in \mathcal{S}, \sigma=\sigma \uplus \mathrm{AI}\left((\operatorname{pre}(\ell), m o, m),\left(\ell_{u l}, m_{u}, m_{u l}\right)\right)$ else $\sigma=$ $\sigma \uplus(\operatorname{pre}(\ell), m o, m)$

For lock instructions $\ell$ : lock $l$, we first perform the pre-processing step to get a list of program states in which no thread has acquired the lock over mutex variable $l$. Second, we apply the interference from all the unlock instructions over mutex variable $l$ in all the other threads to the current program state. This is required to make sure that we are considering all possible reorderings of locked regions. Finally, the lock instruction $\ell$ is appended in the partial order corresponding to the mutex variable $l$. 